\title{
AN IMMERSIVE VIRTUAL REALITY ENVIRONMENT FOR DIAGNOSTIC IMAGING
}

\author{
by

\section{FRANKLIN KING}

A thesis submitted to the

School of Computing

in conformity with the requirements for

the degree of Master of Science

Queen's University

Kingston, Ontario, Canada

August 2015

Copyright (c) Franklin King, 2015 


\begin{abstract}
Advancements in and adoption of consumer virtual reality are currently being propelled by numerous upcoming devices such as the Oculus Rift. Although applications are currently growing around the entertainment field, wide-spread adoption of virtual reality devices opens up the potential for other applications that may have been unfeasible with past implementations of virtual reality. A virtual reality environment may provide an equal or larger screen area than what is provided with the use of multiple conventional displays while remaining comparatively cheaper and more portable.

A virtual reality application for the viewing of multiple image slices was designed using: the Oculus Rift head-mounted display, Unity, and 3D Slicer. Volumes loaded within 3D Slicer are sent to a Unity application that proceeds to render a scene for the Oculus Rift head-mounted display. Users may interact with the images adjusting windowing and leveling using a handheld gamepad controller. Multiple images may be brought closer to the user for detailed inspection.

Application usage was demonstrated with the simultaneous visualization of concurrent slices of a serial CT scan of a patient with a lung nodule. Also demonstrated was the studying of multiple-sclerosis lesion evolution by visualization of a large timeseries MRI dataset. Experiments for validating usage of the virtual reality system for differential diagnosis and remote collaboration were performed.
\end{abstract}




\section{Acknowledgments}

Firstly, I would like to acknowledge my thesis advisor and supervisor Dr. Gabor Fichtinger for providing me with opportunities and support. I would also like to thank my colleagues and friends from the Laboratory for Percutaneous Surgery including Dr. Andras Lasso and Dr. Tamas Ungi.

I would also like to express my gratitude to all that have welcomed and supported me at the Surgical Planning Laboratory in my time at the Brigham and Women's Hospital. Their guidance has been invaluable and I would like to especially thank Dr. Tina Kapur, Dr. Jayender Jagadeesan, and Dr. Steve Pieper.

The involvement of Dr. Paul Shyn and Dr. Sharath Bhagavatula from the Brigham and Women's Hospital has also been instrumental for this work and I am grateful for their help, feedback, and advice.

This work and I were supported by a research agreement between Siemens Healthcare (SY) and Brigham and Womens Hospital. Gabor Fichtinger was funded as a Cancer Care Ontario Research Chair.

Finally, I would like to thank all my friends and family that have supported me when I needed them. 


\section{Contents}

Abstract $\quad$ i

Acknowledgments $\quad$ ii

Contents

List of Tables $\quad$ v

List of Figures $\quad$ vi

Chapter 1: INTRODUCTION 1

1.1 Thesis Objective . . . . . . . . . . . . . . . . . . 1

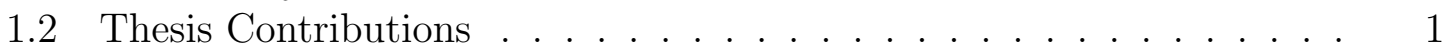

1.3 Thesis Outline . . . . . . . . . . . . . . . . . . 2

Chapter 2: $\quad$ BACKGROUND AND MOTIVATION 3

2.1 Virtual Reality ..................... 3

2.1.1 Virtual Reality Devices . . . . . . . . . . . . . . . . . . 4

2.1.2 Modern Consumer Virtual Reality Devices . . . . . . . . . . . 8

2.2 Applying Virtual Reality to Radiology . . . . . . . . . . . . . . . . . 11

2.2.1 Field of View . . . . . . . . . . . . . . . . . . . . . . . . . . . . . . . . . . . 12

2.2.2 Portability and Cost . . . . . . . . . . . . . . . 13

2.2.3 Remote Collaboration . . . . . . . . . . . . . . . . 14

Chapter 3: PROTOTYPE DESIGN 15

3.1 Overview . . . . . . . . . . . . . . . . . . . 15

3.2 Display . . . . . . . . . . . . . . . . . . . . . 15

3.3 Interface Devices . . . . . . . . . . . . . . . . . . . . . 17

3.3.1 Leap Motion Controller . . . . . . . . . . . . . . . 17

3.3.2 Gamepad Controller . . . . . . . . . . . . . . . . . . 18

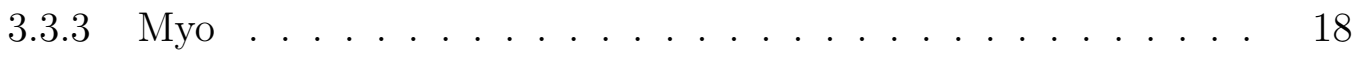

3.4 Software . . . . . . . . . . . . . . . . . . . 19 
3.4 .1 3D Slicer . . . . . . . . . . . . . . . . . . . . . . . 19

3.4 .2 Unity . . . . . . . . . . . . . . . . . . . . . . 20

3.4 .3 System Architecture . . . . . . . . . . . . . . . . 20

3.5 Prototype Usage . . . . . . . . . . . . . . . . . . . . . . . . . . . . 21

3.5.1 Virtual Button Interface . . . . . . . . . . . . . . . . 23

3.5.2 Myo Armband Interface . . . . . . . . . . . . . . . . . . . 24

3.5.3 Gamepad Controller Interface . . . . . . . . . . . . . 25

3.6 Multi-user Support . . . . . . . . . . . . . . . . . . . . . 26

Chapter 4: USE CASES $\quad 27$

4.1 Differential Diagnosis . . . . . . . . . . . . . . . . . . 27

4.2 Remote Instruction and Collaboration . . . . . . . . . . . . . 31

4.3 Visualizing Large Datasets . . . . . . . . . . . . . . . . . . . 31

Chapter 5: EXPERIMENTS AND RESULTS 33

5.1 Differential Diagnosis Experiment . . . . . . . . . . . . . . 33

5.1.1 Differential Diagnosis Experiment Design . . . . . . . . . . . 33

5.1.2 Differential Diagnosis Experiment Results . . . . . . . . . . 35

5.1.3 Differential Diagnosis Experiment Discussion . . . . . . . . . 37

5.2 Remote Collaboration Experiment . . . . . . . . . . . . . 40

5.2.1 Remote Collaboration Experiment Design _ . . . . . . . . . . 40

5.2.2 Remote Collaboration Experiment Results . . . . . . . . . . . 40

5.2.3 Remote Collaboration Experiment Discussion . . . . . . . . 41

Chapter 6: CONCLUSIONS AND FUTURE WORK 42

6.1 Summary of Work . . . . . . . . . . . . . . . . . . . 42

6.2 Future Work . . . . . . . . . . . . . . . . . . . . 43

Bibliography $\quad 45$

Appendix A: Trial Data Forms $\quad \mathbf{5 0}$

A.1 NASA Task Load Index Questionnaire . . . . . . . . . . . . . 51

A.2 Data Sheet . . . . . . . . . . . . . . . . . . 52 


\section{List of Tables}

5.1 Total number of cases assessed by all participants and assessments that were consistent with measured results . . . . . . . . . . . . . 36 


\section{List of Figures}

2.1 Virtuality Spectrum $[21] \ldots \ldots \ldots \ldots$

2.2 Sutherland's Sword of Damocles [30] . . . . . . . . . . . . 4

2.3 NASA Virtual Environment Workstation [13] . . . . . . . . . 5

2.4 VictorMaxx Cybermaxx 2.0 (left) Forte VFX1 (right) . . . . . . . . 6

2.5 Fakespace Labs' BOOM $[4] \ldots \ldots$. . . . . . . . . . . . 7

2.6 CAVE [9] (left)) ImmersaDesk [10] (right) . . . . . . . . . . . . . 8

2.7 Upcoming modern virtual reality displays: Sony Morpheus (left) Oculus Rift Consumer Version (middle), HTC Vive (right) . . . . . . . . 9

2.8 Announcement dates of modern virtual reality devices . . . . . . . . 10

2.9 Release dates and cost (adjusted for inflation) for selected head-mounted displays with head-tracking . . . . . . . . . . . . 11

2.10 Approximate field of view of the Oculus Rift DK2 HMD compared to usage of a conventional display from $50 \mathrm{~cm}$. . . . . . . . . . . . 13

3.1 Oculus Rift Development Kit 2 (left) DK2 infrared markers (right) . . 17

3.2 Leap Motion Controller . . . . . . . . . . . . . . . . . . . 18

3.3 Xbox 360 Gamepad Controller . . . . . . . . . . . . . . . 18

3.4 Myo armband . . . . . . . . . . . . . . . . . . 19

3.5 System architecture . . . . . . . . . . . . . . . . . 21 
3.6 Hands-on positioning using the Leap Motion controller . . . . . . . . 23

3.7 Using virtual buttons to manipulate the virtual environment . . . . . 24

3.8 Manipulating the virtual environment using a Myo armband . . . . . 25

3.9 Using a gamepad controller (left) control layout (right) . . . . . . . . 26

4.1 Storyboard of a session consisting of identifying a lesion on a CT lung image and evaluating its growth in a longitudinal study . . . . . . . . 29

4.2 Definition of lateral head facing for Figure 4.3 . . . . . . . . . . . . . 30

4.3 Recorded lateral head facing over the course of the storyboard session 30

4.4 Master user locates a lesion and highlights it with a finger . . . . . . 31

4.5 Visualization of a large number of image datasets . . . . . . . . . . . 32

5.1 Diameter and cross-sectional measurements of lung lesions . . . . . . 34

5.2 Average time taken for identification of the largest lung lesion per dataset. Error bars are standard deviation of the mean. . . . . . . . . 37

5.3 Average time taken for growth assessment of the largest lung lesion per dataset. Error bars are standard deviation of the mean. . . . . . 38

5.4 NASA TLX for VR and standard DICOM viewer usage. Error bars are for standard error. . . . . . . . . . . . . . . . . . . . . . . 38

5.5 NASA TLX for remote collaboration. Error bars are for standard error. 41

6.1 Stereoscopic video support (left) Tractography fiber bundles viewed in virtual reality (right) . . . . . . . . . . . . . . . . 44 


\section{Chapter 1}

\section{INTRODUCTION}

\subsection{Thesis Objective}

Our goal for this work was to apply the advent of a new generation of consumeroriented immersive virtual reality devices to the field of diagnostic imaging. Current radiological practice commonly involves the use of dedicated radiology workstations and reading environments. Substituting or augmenting the use of these physical reading environments with a virtual environment may provide benefits involving usage of space, accessibility, and remote collaboration.

We seek to demonstrate that modern consumer virtual reality devices are able to provide the image quality necessary to perform radiological tasks. We hope this work also demonstrates the potential of a low-cost virtual reality environment in improving or providing additional benefits to the radiological practice.

\subsection{Thesis Contributions}

- Developed a virtual reality radiology reading room environment prototype

- Explored multiple interface concepts for interacting with a virtual reading room 
- Demonstrated use cases for the virtual reality environment

- Validated the use of the virtual reality environment for differential diagnosis and remote collaboration

\subsection{Thesis Outline}

- Chapter 2 Background and Motivation: contains a brief history of virtual reality and consumer virtual reality displays. Motivations for the work and potential benefits of modern virtual reality displays for radiological practice will also be discussed.

- Chapter 3 Prototype Design: details the components and operation of the virtual reality diagnostic imaging environment. Multiple interface systems that were experimented with over the course of development will also be highlighted.

- Chapter 4 Use Cases: demonstrates use cases for the virtual reality diagnostic imaging environment.

- Chapter 5 Experiments and Results: explains the trials performed to evaluate the usage of the prototype for differential diagnosis and remote collaboration and delivers the results obtained.

- Chapter 6 Conclusions and Future Work: summarizes the work performed and discusses future applications of the technology within radiology and related practices. 


\section{Chapter 2}

\section{BACKGROUND AND MOTIVATION}

\subsection{Virtual Reality}

Virtual reality is an artificial environment that is created to be experienced with and interacted by a person. Although existing together on a spectrum depicted in Figure 2.1 , virtual reality is distinguished from augmented reality in that the intention of virtual reality is for a user to be totally immersed within an artificial environment $[21]$.

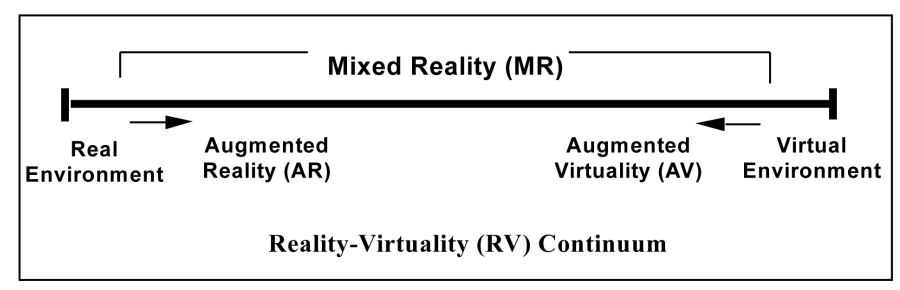

Figure 2.1: Virtuality Spectrum [21]

Virtual reality has long been explored as a technology for creating immersive environments conducive to scientific visualization [5]. Historically, virtual reality systems have attempted to accomplish this using technologies such as head or vehicle tracking, large field of view displays, and stereoscopic imaging. Past work in the 
area of virtual reality dates as far back as 1968 with what is often considered the first virtual reality head-mounted display by Sutherland [30]. Sutherland's "Sword of Damocles" pictured in Figure 2.2 displayed wireframe graphics with a perspective determined by head-tracking accomplished with a ceiling mounted mechanical arm.

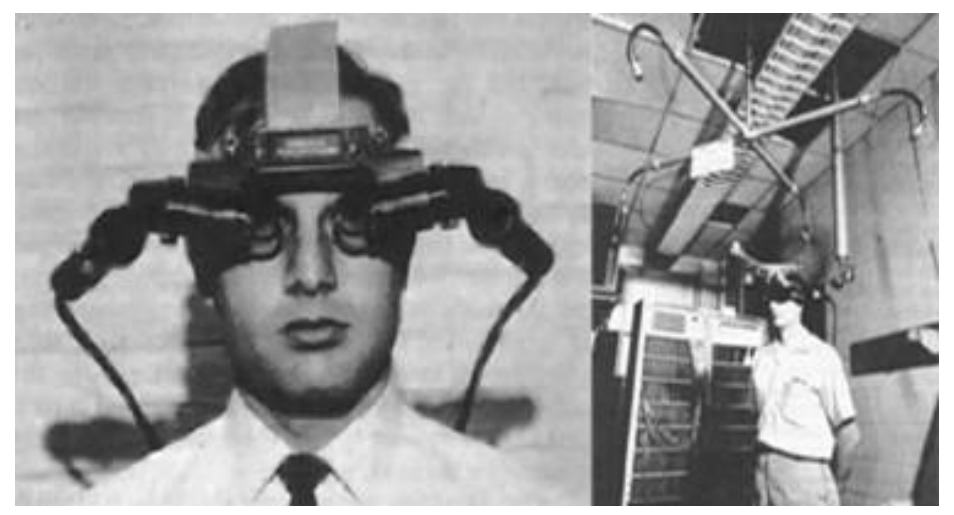

Figure 2.2: Sutherland's Sword of Damocles [30]

\subsubsection{Virtual Reality Devices}

Development of virtual reality (VR) technology had continued since the experiments with the "Sword of Damocles" albeit initially restricted to multi-million dollar systems intended for military or otherwise non-personal use. Later systems such as NASA's Virtual Environment Workstation in 1984 (Figure 2.3), aside from coining the term "virtual reality," made progress towards consumer affordable virtual reality [13]. It wasn't until later, however, that consumer-oriented VR devices were made available in the consumer market.

Consumer-oriented VR devices have been available since the late 1980s but with limited success in consumer adoption for practical applications. Multiple technologies are crucial for satisfactory VR experiences including display resolution, tracking systems, interfaces, latency, and graphics rendering. Other important factors for a 
successful consumer-oriented device are cost, portability, and other comfort and convenience factors. Widespread consumer adoption had been limited due to deficiencies in all or some of these areas. Most notably, cost and lack of significant software support. Without widespread consumer adoption, any potential practical application for VR must justify the often significant cost of a VR device.
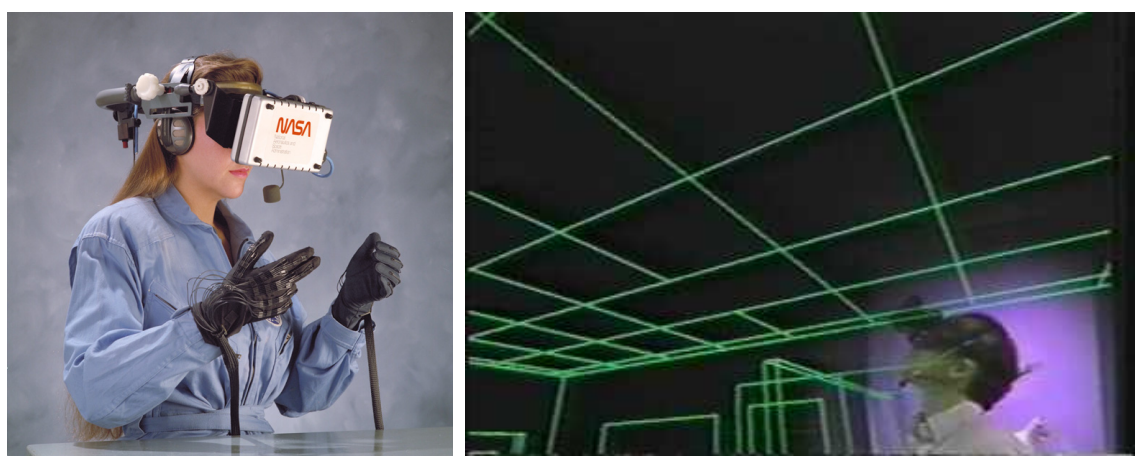

Figure 2.3: NASA Virtual Environment Workstation [13]

Various virtual reality systems have been developed and demonstrated for a wide variety of applications in the past, including medical applications. Pieper et al. [26] have demonstrated usage of a head-tracked stereo display for minimally invasive surgery in 1994. Calvano et al. [6] have also investigated using a head-mounted display for real-time 3D imaging for endoscopic fetal surgery. Krapichler et al. [19] presented medical imaging applications using a virtual reality environment including virtual bronchoscopy and virtual angioscopy in 1998. Widespread adoption of immersive virtual reality devices for medical applications, however, has not occurred possibly due to various previously mentioned reasons such as high cost and inadequate interface systems. 


\section{Head-mounted LCD Displays}

One example of an early consumer VR head-mounted display device was the VictorMaxx CyberMaxx 2.0 released in 1995. The CyberMaxx 2.0 features usage of LCD panels and head-tracking and has a form factor reminiscent of more modern VR devices. With a resolution of $263 \times 230$ pixels per eye and a horizontal field of view of 54 degrees, it suffered from poor image quality with details such as text being hard to read and an initial retail price of $\$ 889$ USD (\$1402 USD today adjusted for inflation $\left.^{1}\right)$. Another example was the Forte VFX1 also released in 1995 retailing initially for $\$ 995$ USD (\$1553 USD today adjusted for inflation). Featuring The VFX1 and other contemporary displays suffered from the same issues with poor resolution, latency, and cost [24].
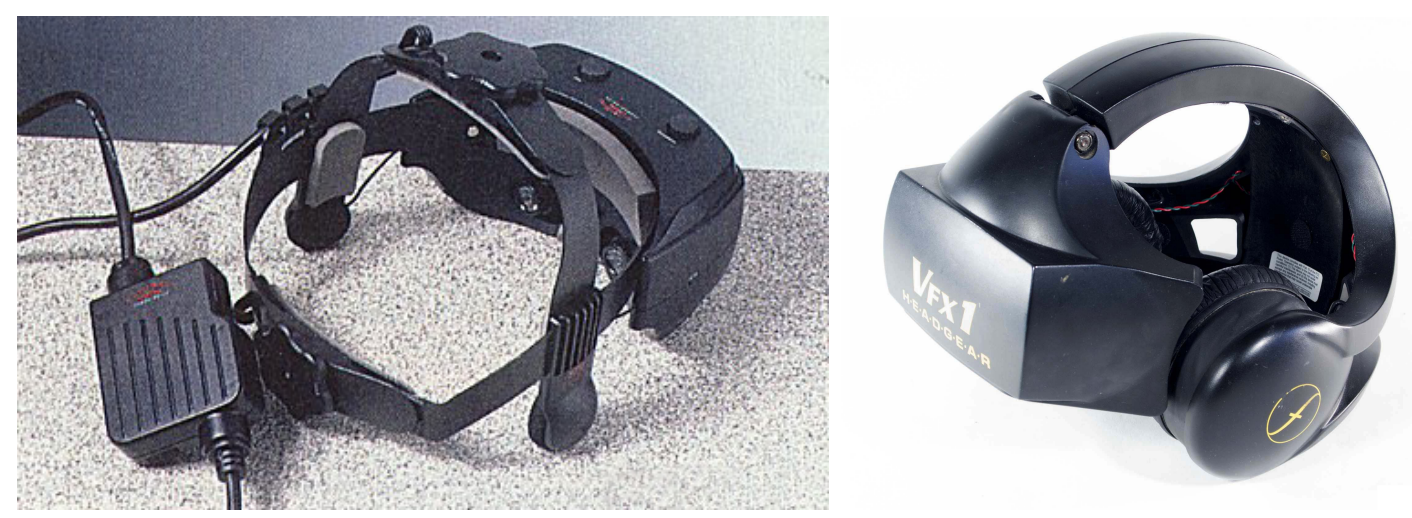

Figure 2.4: VictorMaxx Cybermaxx 2.0 (left) Forte VFX1 (right)

\section{BOOM Display}

The BOOM (Binocular Omni-Orientation Monitor) created by Fakespace Labs was an example of an system intended to address perceived problems with contemporary head-mounted displays [4]. It and other devices relying on CRT displays exhibited

\footnotetext{
${ }^{1}$ http://www.bls.gov/data/inflation_calculator.htm
} 
improved image quality, but were cumbersome and often significantly more expensive. The BOOM made use of a mechanical tracking system again due to deficiencies with magnetically tracked contemporary head-mounted displays. The use of sensors within a counterbalanced arm led to a large system that required a significant amount of space for setup as seen in Figure 2.5.

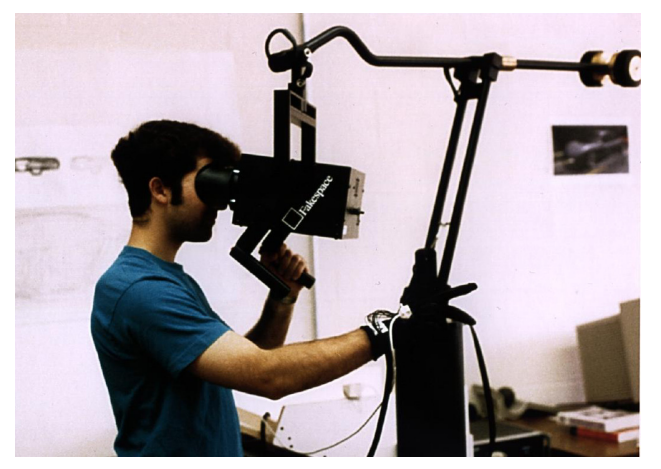

Figure 2.5: Fakespace Labs' BOOM [4]

\section{CAVE and CAVE-derived Displays}

The CAVE consisted of multiple projectors directed to the walls and floor of a room [9]. It supported head-tracking and the viewing of stereoscopic images through the use of liquid crystal shutter glasses. The CAVE was originally developed in order to provide a large field of view for scientific applications while also allowing for collaboration between multiple participants in a VR environment. Although it overcomes the limitations of head-mounted displays of its time, usage of a CAVE requires large amount of space for setup, in comparison to head-mounted displays, and suffers in regards portability and ease of setup.

Developed in 1994, the ImmersaDesk [10] was a CAVE-derived VR display featuring a 170x127 cm rear-projected screen tilted at a 45 degree angle featuring stereoscopic, head-tracked images. Like the CAVE, it allowed for multiple users to interact 
with the environment although only one user's head is tracked. Significantly more portable than the CAVE, the ImmersaDesk still had a footprint of approximately $86 \times 185 \mathrm{~cm}$ while folded in its transportation position.
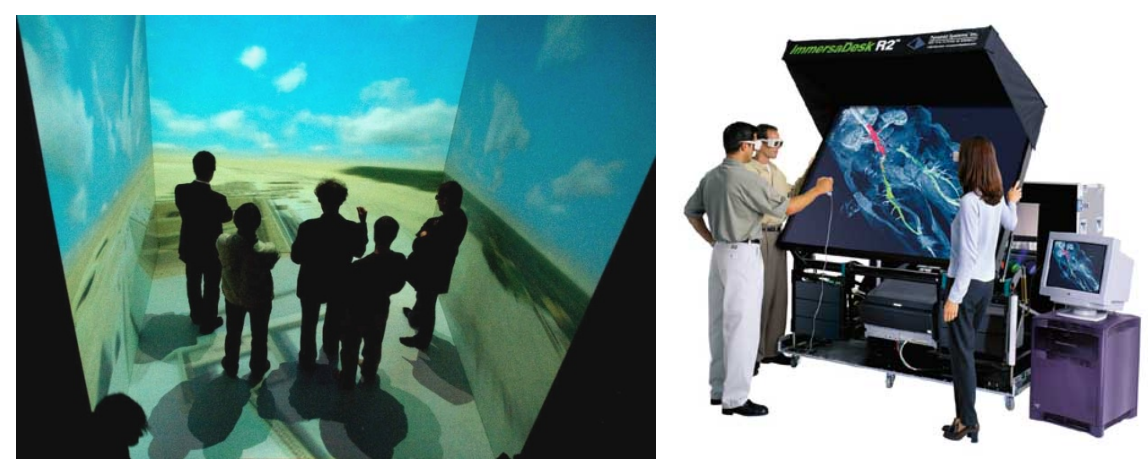

Figure 2.6: CAVE [9] (left)) ImmersaDesk [10] (right)

\subsubsection{Modern Consumer Virtual Reality Devices}

By the mid-1990s the idea that the virtual reality bubble had ended entered the public consciousness [20]. Recently, however, there has been a resurging interest in affordable, consumer virtual reality systems. Notable technologies important to VR have manifested in modern smartphone development while computing technology for real-time 3D rendering has improved notably in the realm of video games. Portable and relatively cheap high-resolution displays and motion sensors have led to the development of new VR devices such as the Oculus Rift, introduced by Oculus VR (Irvine, CA )[33] in late 2012 and acquired by Facebook, inc. for $\$ 2$ billion USD in 2014, the Sony Morpheus, and HTC Vive all depicted in Figure 2.7. Other companies developing VR and mixed reality devices include Microsoft and Samsung. Figure 2.8 is a listing of virtual reality devices that have been announced since the 2012 announcement and crowd-funding campaign of the Oculus Rift ${ }^{2}$. Although

\footnotetext{
${ }^{2}$ http://www.kzero.co.uk/blog/category/virtual-reality/
} 
current applications for these new consumer-oriented VR head-mounted displays are centered around the entertainment field, specifically the video game industry, potential widespread adoption of VR devices makes practical other applications that beforehand would have required specialized and potentially expensive hardware.

Head-mounted displays are now overcoming limitations in weight, accuracy, and cost that have previously precluded their practical use. The current developer model of the Oculus Rift HMD weighs 440g with the consumer model not expected to weigh significantly heavier. The consumer model is also set to retail at approximately $\$ 300$ USD. Figure 2.9 is a non-comprehensive chart of the release dates and initial retail cost (adjusted for inflation ${ }^{3}$ for past release dates) of selected head-mounted and head-tracked virtual reality displays as of July 2015.
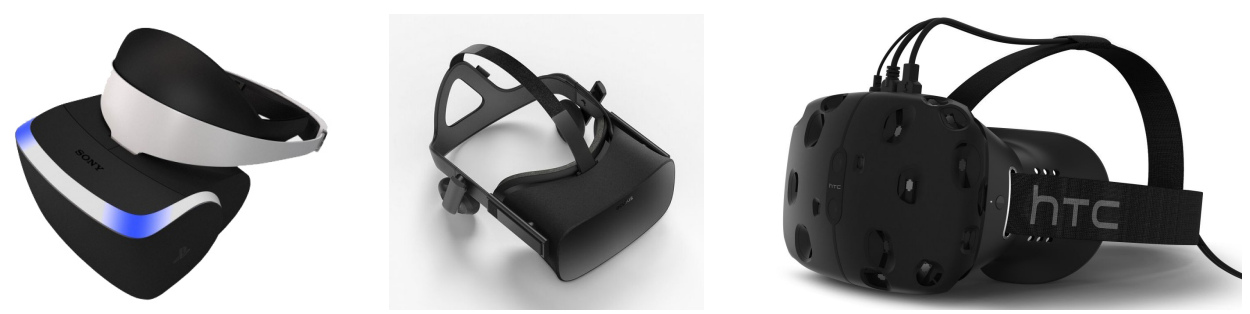

Figure 2.7: Upcoming modern virtual reality displays: Sony Morpheus (left) Oculus Rift Consumer Version (middle), HTC Vive (right)

\footnotetext{
${ }^{3}$ http://www.bls.gov/data/inflation_calculator.htm
} 


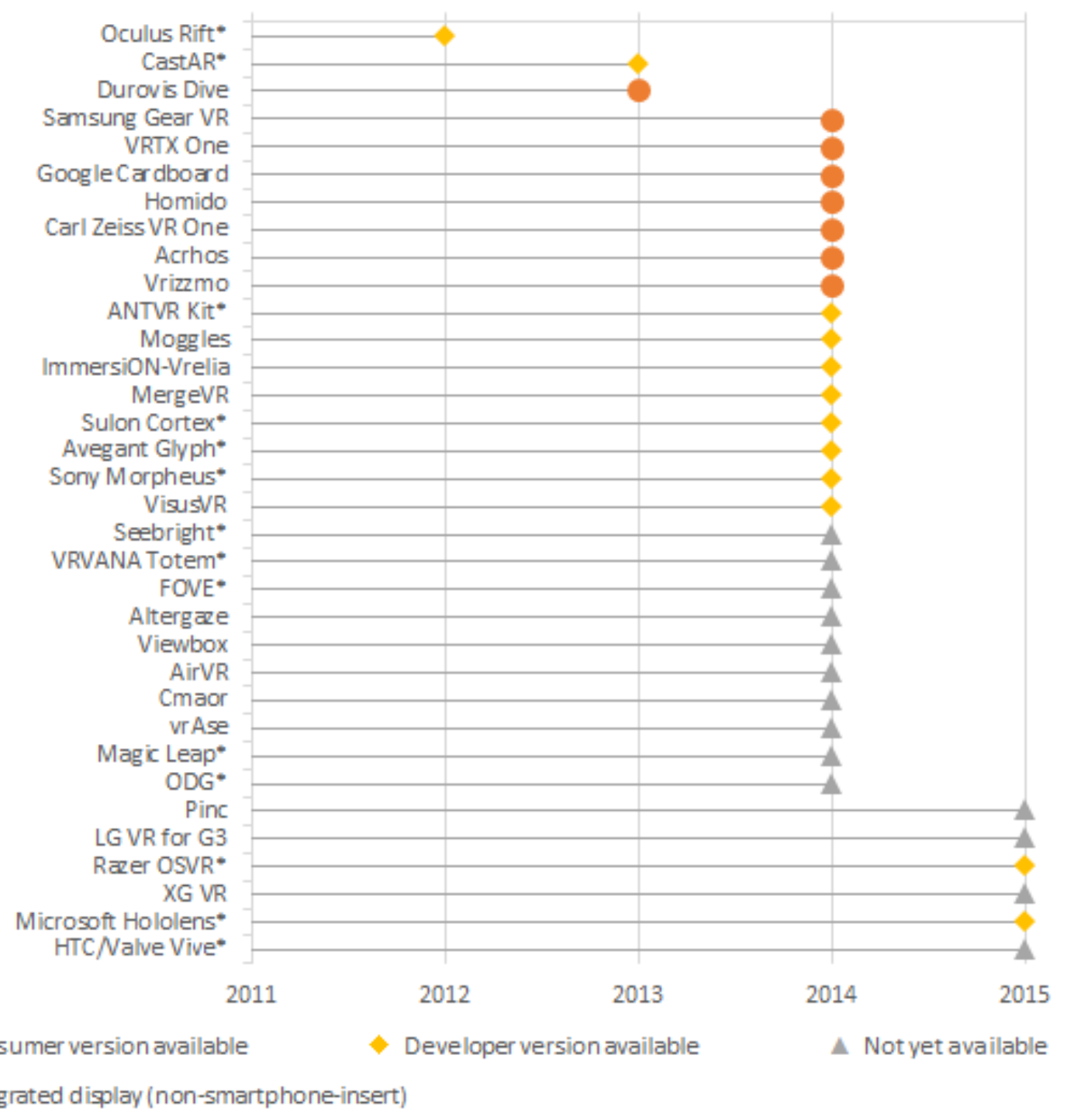

Figure 2.8: Announcement dates of modern virtual reality devices 


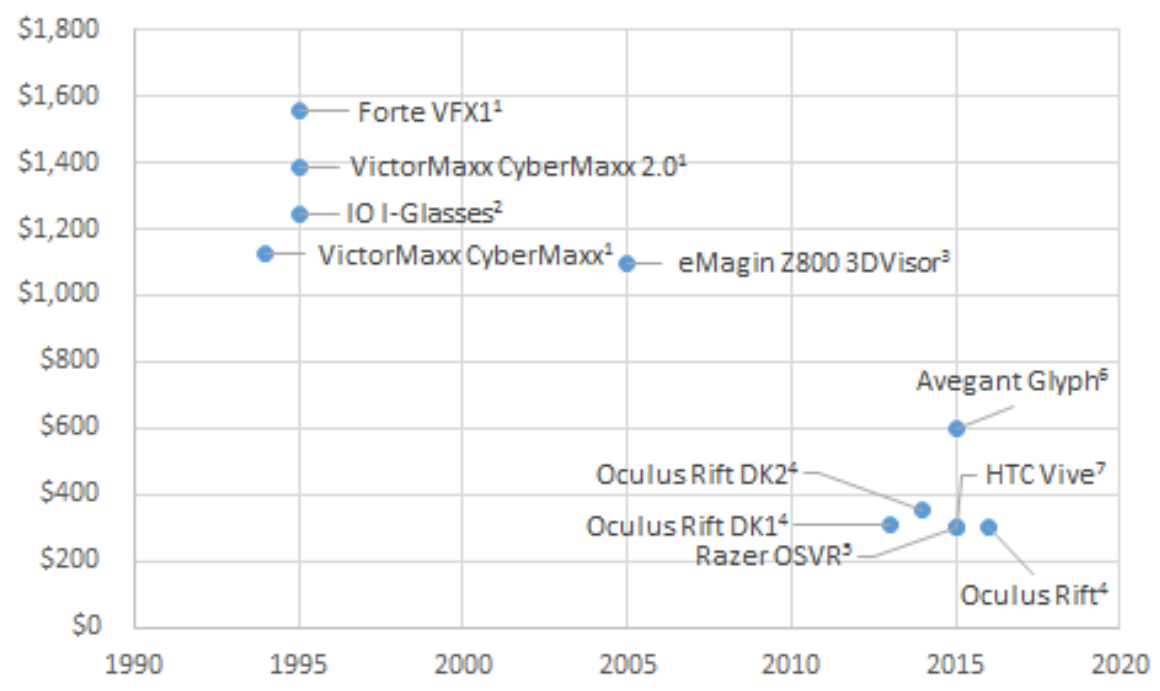

Figure 2.9: Release dates and cost (adjusted for inflation) for selected head-mounted displays with head-tracking

\subsection{Applying Virtual Reality to Radiology}

Radiologists currently spend a significant amount of time interpreting medical images from computer workstations in picture archiving and communication system (PACS) reading rooms. The adoption of PACS reading rooms has led to, for the most part, improved efficiency and decreased use of space compared to the use of film [23]. However, surveys of radiologists such as those performed by Rumreich \& Johnson [28] have highlighted issues involving poor lighting, insufficient space, and few accommodations for large multi-monitor workstations. Similarly, a survey by Siegel et al. [29]

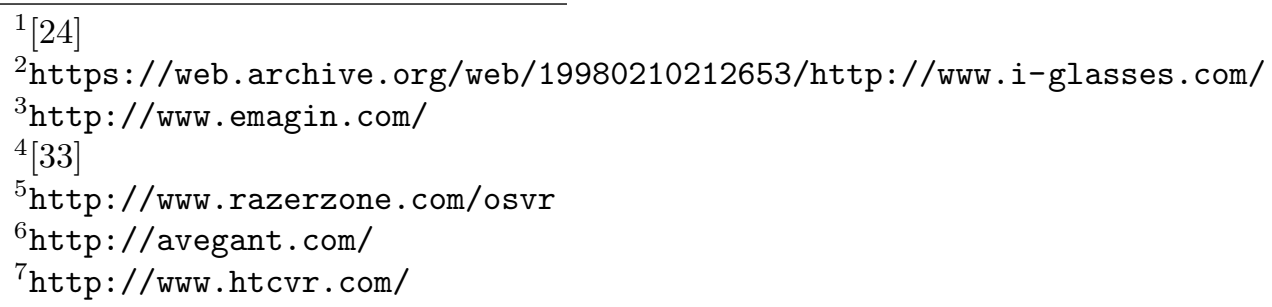


found some of the most important factors to radiologist productivity to be ambient room lighting and monitor number. The advent of modern head-mounted VR devices may provide a potential solution to some of the current concerns and issues faced by radiologists regarding the usage of PACS reading rooms.

\subsubsection{Field of View}

Virtual reality displays offer a large field of view as demonstrated in Fig. 2 that can only be obtained traditionally when using multiple conventional flat displays or a singular large display. Large displays have been shown to act as a benefit in regards to tasks such as those involving visual scanning and in regards to both 3D and $2 \mathrm{D}$ data [25][3][32]. In addition to this, it is possible that the visceral illusion of being within a simulated environment, also known as presence, may in of itself contribute as a psychological benefit to improved performance on visual tasks [34]. Head-tracking allows for a user to look around an environment in a manner more natural and intuitive when compared to scrolling on traditional displays. A virtual reality radiology reading room environment can thus be considered analogous to large multi-monitor workstations with a practically limitless number of monitors that may be added or removed. 


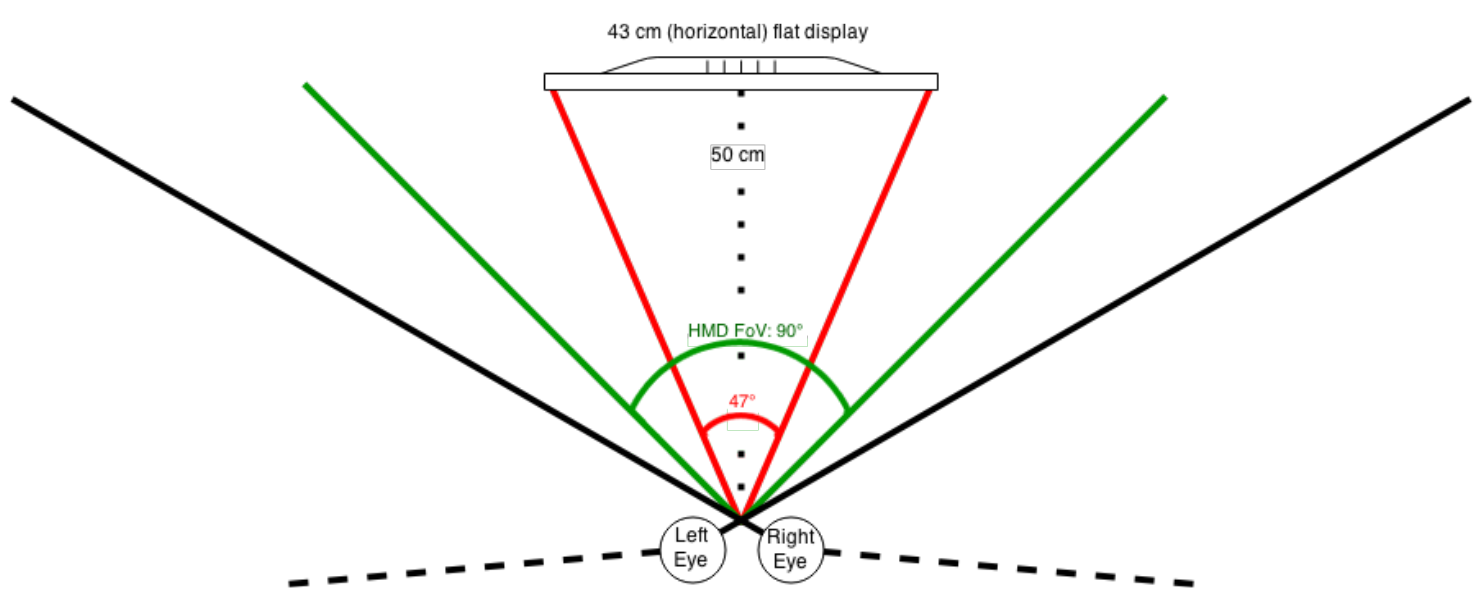

Figure 2.10: Approximate field of view of the Oculus Rift DK2 HMD compared to usage of a conventional display from $50 \mathrm{~cm}$

\subsubsection{Portability and Cost}

The design of a standard radiology reading room aids the comparison aspect of such tasks through the use of multiple conventional flat displays. Increasing the monitor number of a traditional reading room requires an investment of space and cost that can prove prohibitive to some sites unlike the case with a virtual reading room. Issues involving ambient lighting may also be addressed when a radiologist has access to a personal virtual reading room rather than sharing a space with other users. Additionally, the use of a virtual reality environment may allow for diagnostic radiology tasks to be completed in a virtual reading room accessible to those without access to a standard reading room as may be the case in rural areas or areas otherwise outside of a hospital setting. 


\subsubsection{Remote Collaboration}

Ai et al. [1] made use of the aforementioned CAVE and ImmersaDesk displays for loading medical data into a tele-immersive environment allowing for geographically distributed clinicians to point and gesture with each other. Such collaborative actions could be replicated with modern VR displays such as the Oculus Rift with a lower cost and space requirement. Unlike CAVE displays, a head-mounted VR system may be used for remote collaboration without requiring all participating users to be present in the same physical space. Also unlike CAVE displays, head-tracking functionality is not limited to a single participant but instead given to each user. 


\section{Chapter 3}

\section{PROTOTYPE DESIGN}

\subsection{Overview}

In this chapter we describe the design of our virtual reality diagnostic imaging environment prototype and the multiple implementations that were experimented with over the course of development. The main components of the prototype consist of a head-mounted display device, the interface device, and the architecture for the loading and manipulation of image datasets.

\subsection{Display}

The Oculus Rift Development Kit 2 (DK2) was chosen as the virtual reality headmounted display to be used for the prototype. The DK2 was the second iteration of the Oculus Rift released publicly by Oculus VR [33] in 2014 and was intended to be available ahead of the consumer model in order to allow developers to begin integrating the device for applications.

Contained within the DK2 is a 1920x1080 pixels (960x1080 pixels per eye) OLED panel that is viewable through dual lenses that magnify the image giving a user a 
stereoscopic 3D perspective with a wide field of view of approximately 90, greater than the field of view gained from standard usage of a tablet or smartphone display. However, the lenses impart a significant pincushion distortion to the image, therefore applications designed for the Oculus Rift output a barrel distorted image which is then canceled out by the lens distortion. In addition to correcting for image distortion, software also must correct for chromatic aberration caused by the lenses. The DK2 also includes multiple lenses that correct for near-sightedness when using the device. The resolution of the DK2 is not sufficient enough to completely avoid what is known as the "screen door effect", that is, the ability to notice the individual pixels of the display. Another particular interest is the usage of a low persistence display. Unlike a traditional display, the DK2's panel pixels only light for $2 \mathrm{~ms}$ a frame. Combined with a refresh rate of $75 \mathrm{~Hz}$, the result is reduced motion blur experienced by the user allowing for smaller details to be viewable to a user such as text. Such readability may be of significant importance for tasks requiring a user to analyze subtle changes between multiple images.

The DK2 features both rotational and positional head tracking with 6 degrees of freedom and sub-millimeter accuracy. Rotational tracking is accomplished using a combination of gyroscope, accelerometer, and a magnetometer to correct for drift. Positional tracking is achieved using an external near infrared camera and a series of blinking infrared LEDs positioned within the DK2's infrared-transparent housing viewable in Figure 3.1.

As mentioned in Chapter 1, the DK2 weighs $440 \mathrm{~g}$ and had a cost of $\$ 350$ USD. As of this time, the consumer model (CV1) was announced to be available in early 2016. An improvement in resolution to $2160 \times 1200$ pixels (1080x1200 pixels per eye) 
is expected among other improvements to aspects such as tracking.
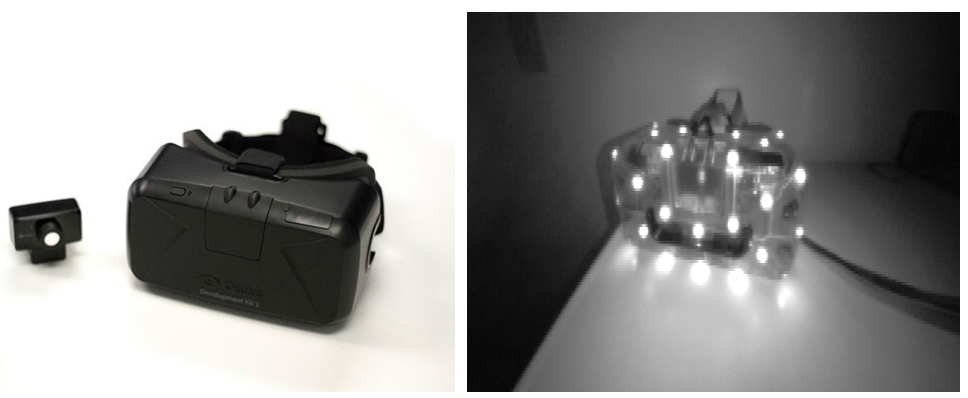

Figure 3.1: Oculus Rift Development Kit 2 (left) DK2 infrared markers (right)

\subsection{Interface Devices}

In order for the user to accomplish certain actions within the prototype, such as scrolling through images and adjusting image settings, an interface device is needed. Aside from a mouse and keyboard, the following devices were implemented for usage with the prototype.

\subsubsection{Leap Motion Controller}

The Leap Motion controller [7] is a small device designed to perform touchless hand and finger recognition in an approximately 1 meter radius hemispherical area. It consists of two monochromatic infrared cameras and three pattern-less infrared LEDs. The Leap Motion controller was mounted to the front of the Oculus Rift DK2 using Leap Motion's VR developer mount. When mounted to the DK2 in such a manner the Leap Motion controller's cameras may be used as a passthrough camera allowing a user to view their physical surroundings. 
Figure 3.2: Leap Motion Controller

\subsubsection{Gamepad Controller}

Another interface device considered was a gamepad controller. In this case, the gamepad controller used was a Wireless Microsoft Xbox 360 Controller [8]. The Xbox 360 controller features 2 analog joysticks, 2 analog triggers, and 11 digital buttons. A radio receiver was used to connect the controller to a workstation. Clinicians may be unfamiliar with the layout of the gamepad controller requiring a small amount of time for familiarization.

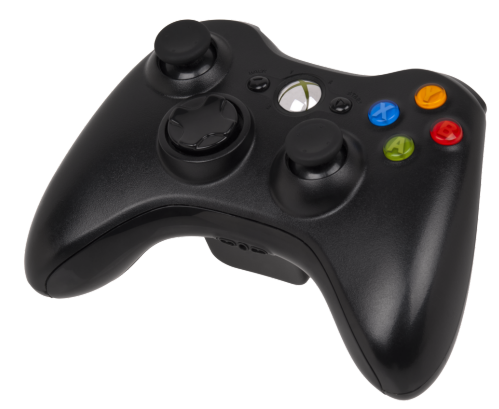

Figure 3.3: Xbox 360 Gamepad Controller

\subsubsection{Myo}

The Myo [22] is a wireless armband created by Thalmic Labs that is intended for gesture control. It accomplishes gesture control using multiple electromyography 
(EMG) sensors that are fitted around the user's forearm along with a gyroscope, accelerometer, and magnetometer. Gestures recognized by the Myo include waves, fists, and spread fingers. Unlike other hand tracking solutions such as the Leap Motion controller, the Myo suffers from a limited range of gestures and requires significant time for calibration.

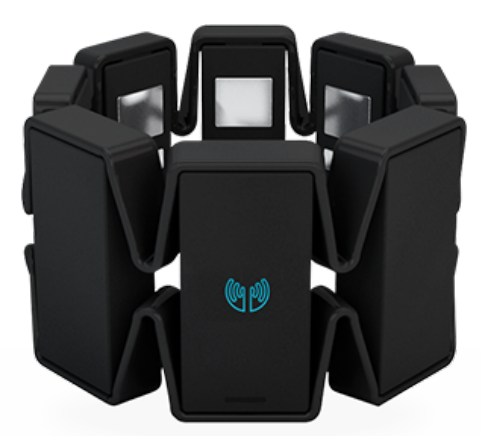

Figure 3.4: Myo armband

\subsection{Software}

The virtual reality diagnostic imaging system uses both 3D Slicer and Unity software packages. 3D Slicer is used to handle the loading and visualization of medical DICOM image data whereas Unity is used to construct the virtual reality environment to be viewed with the DK2.

\subsubsection{D Slicer}

3D Slicer is a free, comprehensive, and open-source software package widely used for a variety of medical applications including image-guided interventions [12]. Among its features are support of numerous common data formats, a number of state-of-the-art image registration algorithms, ease of use, and comprehensive data visualization. The 
application features a modular organization, allowing for the easy addition of plugins known as modules. Accordingly, 3D Slicer was chosen as the platform for the loading and visualization of medical image datasets.

Making use of 3D Slicer's modularity, a web server module was created and used to forward volume slice image data to the virtual reality application. Commands could also be sent to the 3D Slicer web server allowing for control over 3D Slicer's functions.

\subsubsection{Unity}

Unity [11] is a video game engine and development toolkit maintained and developed by Unity Technologies. As a software framework intended for video game development, it features 2D and 3D renderers and support for physics, collision detection, animation, networking, memory management, threading, and etc. Unity is one of currently two game engines that Oculus VR directly supports integration of the Oculus Rift for. Using Unity allowed for smooth integration of display and interface devices while maintaining a focus on high frame rate and allowing for easier implementation of additional functionality such as networked multi-user usage.

\subsubsection{System Architecture}

The architecture is laid out in Figure 3.5. Loading of DICOM image data is first handled by 3D Slicer which then creates a display for each image volume viewable in a slice window with set windowing and leveling. DICOM metadata is also parsed and displayed as annotations overlaid on 3D Slicer's image slice windows. The Unity application creates a scene and queries the 3D Slicer Web Server module for the 
image slice windows which are then displayed and arrayed within the Unity scene. User commands affecting images are sent to the Web Server module which sends back an updated image. The Unity application also renders the scene stereoscopically with distortion and chromatic aberration. The application also makes use of the DK2's "Direct-To-Rift" mode allowing for usage of a custom kernel driver to write directly to the DK2 reducing latency and providing a framerate of 75 FPS for the DK's 75 $\mathrm{Hz}$ display among other benefits. The prototype setup was designed for exploratory purposes and is currently specific for the hardware used. In future, a generic interface allowing for usage with multiple other VR displays and interfaces may be developed.

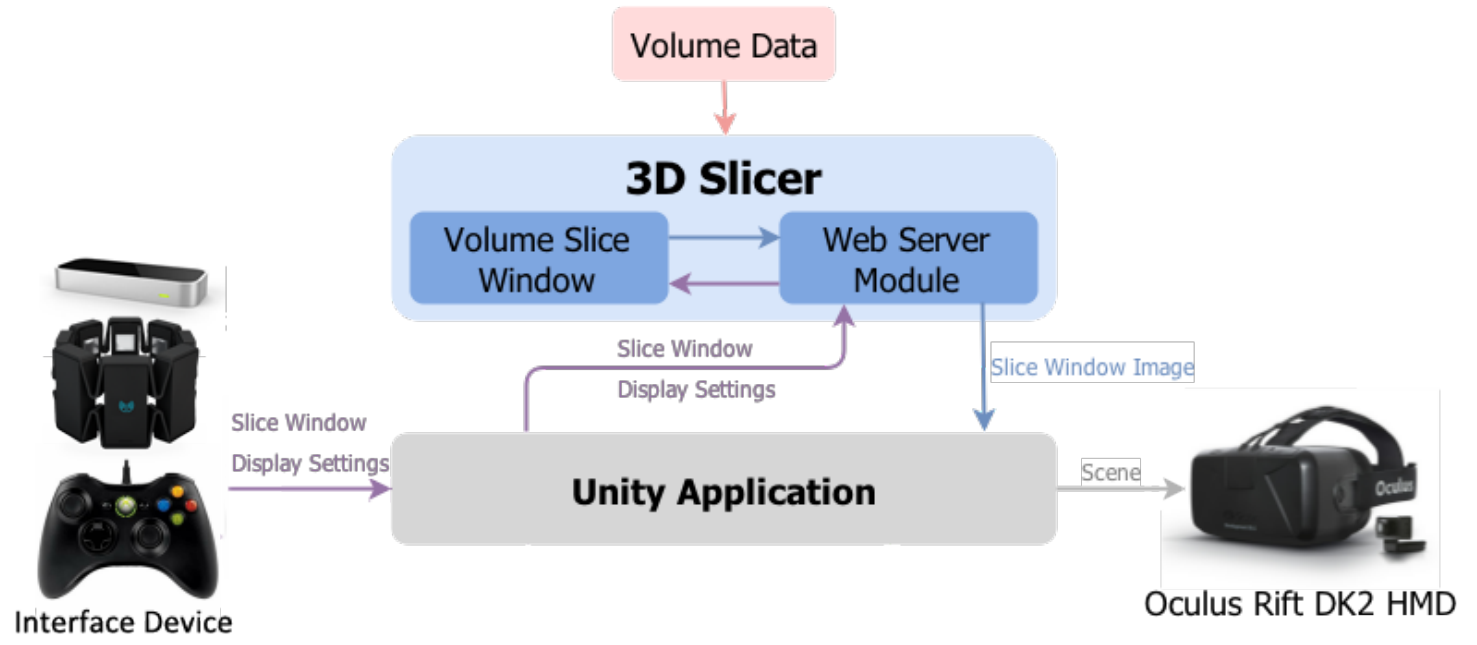

Figure 3.5: System architecture

\subsection{Prototype Usage}

The virtual reality diagnostic imaging prototype created is capable of displaying, as a slice window, any volume from any image modality that can be loaded in 3D Slicer. The stereoscopic display of the DK2 is used to create an immersive environment for 
a user and the display of stereocopic images, volume renderings, and 3D models were not yet explored within the scope of this work. At startup, image datasets are arrayed at a distance allowing for a quick preview of the image content, but not at the detail required for in-depth examination. At this point, users have multiple actions available for manipulation of the images. Placing the view reticule visible at the center of the user's field of view over an image displays an overlay showing the date of the study the image originated from. Scanning over multiple images this way may allow for a user to pick out a desired image set taken at a specific date. Using an interface device to select an image brings the image window closer to the user at a distance and image quality that should allow for in-depth examination. Multiple image sets being selected creates a cylindrical array of images around the user conducive to comparing images within longitudinal studies. Comparisons between images over time can be made with intuitive head motions in a way analogous to the usage of multiple traditional computer displays. A closer view can be accomplished by simply leaning towards an image. The user may also scroll through each image volume and adjust the windowing and leveling settings. Only the image that the user is looking at is affected as determined by the position of the reticule. If desired, the ability to walk around the scene is also available. Using the mounted Leap Motion controller, users can also manually adjust the position and orientation of images in a hands-on manner as seen in Figure 3.6. A button or gesture is held in order to allow for images to stick to the hand of the user. The image is left in place when the button is let go or the gesture is stopped. Pressing the selection button or performing the selection gesture returns the image to its default position. Other actions such as adding annotations or dictations are not implemented. 
Several implementations were experimented with using the interface devices available in order to allow for the user to accomplish the interactions described. Traditional mouse and keyboard interfaces can prove suboptimal to use in a virtual reality environment as it both constrains one's ability to move freely and increases the risk of losing track of the position of one's hands over the keyboard. For general use among clinicians, other interface devices had to be considered.

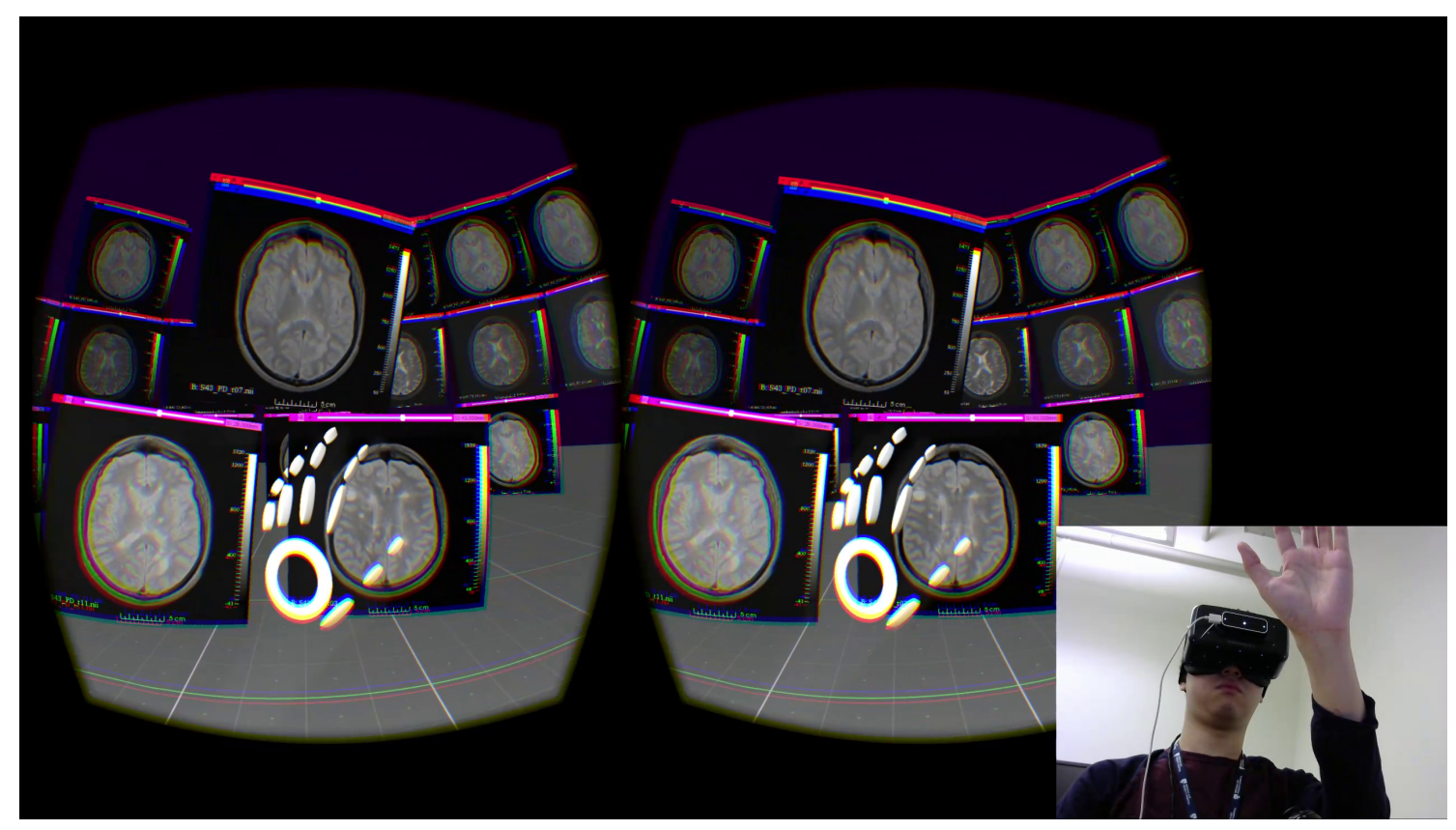

Figure 3.6: Hands-on positioning using the Leap Motion controller

\subsubsection{Virtual Button Interface}

Using the Leap Motion controller it is possible to display a virtual hand model in a manner consistent with the movement of the user's hands in reality. With this in mind, virtual buttons were added to the prototype environment allowing for the user to manipulate images using their hands as seen in Figure 3.7. Although the usage of virtual buttons may be intuitive in a virtual environment, multiple obstacles led 
to usage of another interface system. Such obstacles included the chance of the Leap Motion Controller losing track of a user's hand especially when close to a surface, the blocking of the DK2's positional tracking camera with a hand, the lack of haptic feedback, and an issue colloquially termed the gorilla-arm effect. The gorilla-arm effect is the issue of fatigue occurring from extended usage of a gestural interface without allowing a user to rest [16][14]. Without an overriding reason to primarily use mid-air interactions for radiology tasks, we deemed the fatigue and strain arising from such interactions to require another interface design for the virtual reality diagnostic imaging system.

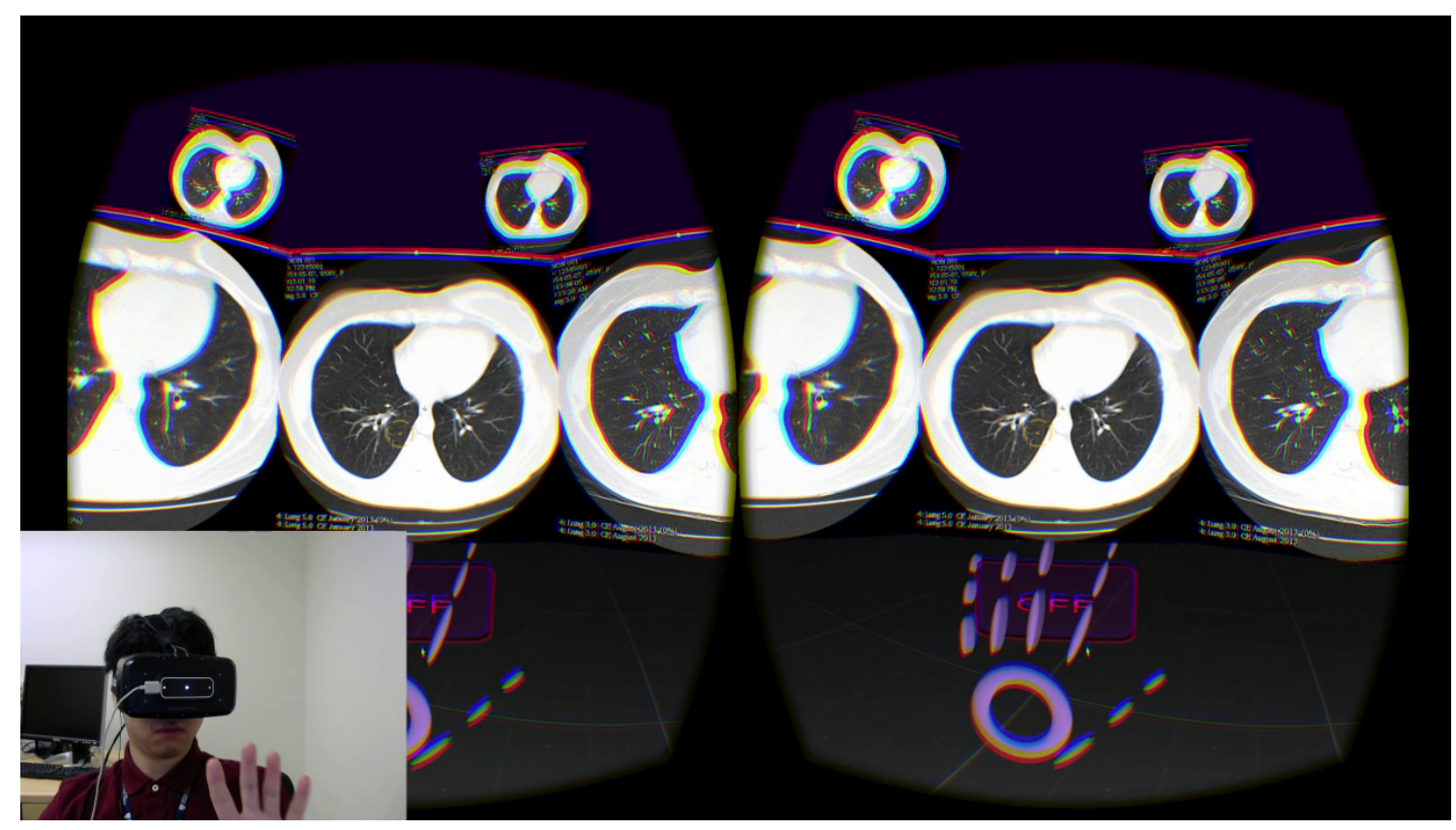

Figure 3.7: Using virtual buttons to manipulate the virtual environment

\subsubsection{Myo Armband Interface}

The Myo armband allows for touch-free interaction without the need for a camera to keep track of a user's hands. Actions were mapped to specific gestures such as the 
closing of a fist or the spreading of fingers. Figure 3.8 shows a closing fist gesture being used to select an image. Ultimately, achieving reliable performance with the Myo required a calibration to be performed each time the armband is adjusted or removed. Combined with the possibility that gestures could still be recognized incorrectly, a more traditional interface solution was decided upon.

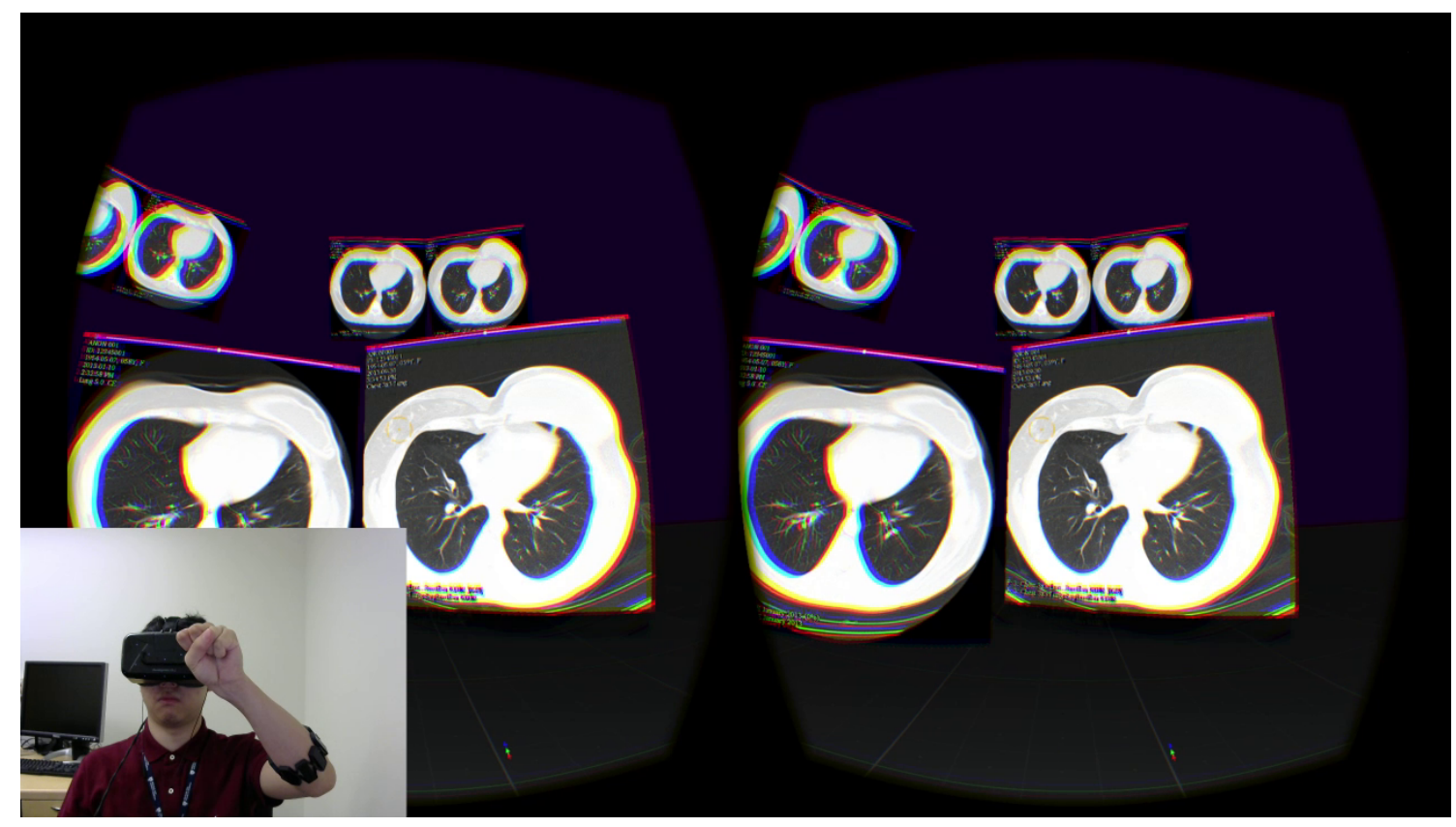

Figure 3.8: Manipulating the virtual environment using a Myo armband

\subsubsection{Gamepad Controller Interface}

The Xbox 360 Gamepad allows for wireless interaction with both analog and digital controls. Windowing and leveling actions were mapped to the two axes of one joystick. Movement was mapped to the other joystick. And scrolling functionality was mapped to the trigger axis. Having these actions on analog controls allowed for a user to control the magnitude of the change desired. Image selection was mapped to the digital buttons. Training time required for using the gamepad was significantly lower 
than what was required with gesture-based controls and the overall ease-of-use led to it being used as the default interface device for the prototype.
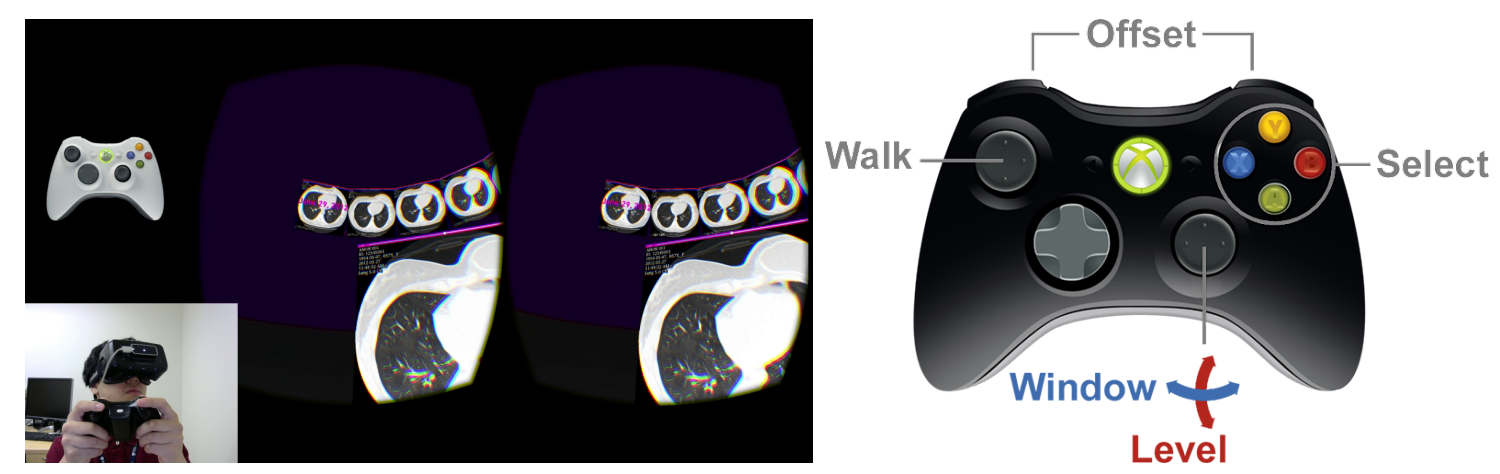

Figure 3.9: Using a gamepad controller (left) control layout (right)

\subsection{Multi-user Support}

The VDRI system supports multiple users viewing and interacting simultaneously with a single scene. Using a master and slave system, all commands performed by the master are copied to the scenes of each slave user. Interaction involving the manipulation of images is also relayed across all users as each user queries the 3D Slicer server for updated images. Hand recognition and movement is also relayed across each user allowing for users to point out areas of interest in a hands-on manner. The master user's reticule position is also visible to all slave users informing them of where the gaze of the master user is directed towards. 


\section{Chapter 4}

\section{USE CASES}

\subsection{Differential Diagnosis}

Due to the potentially poor accuracy of lung biopsies [18], differential diagnosis of lesions is typically performed by monitoring the size of lung lesions over time. In some cases, as many as 7 to 8 scans taken over a two year period are necessary to determine the growth of the lung lesion or the solid component within the lesion. Therefore it is imperative to simultaneously view multiple CT scans to determine the malignancy status by visually detecting a change in size or nature of the solid component of the lesion.

Shown in Figure 4.1 is an example of usage of the virtual reality diagnostic imaging system. Depicted is the simultaneous visualization of 6 CT volumes of a 61 year old patient obtained over a 2 year period loaded in from 3D Slicer. In addition to the images themselves, DICOM metadata which would patient and study information is visible along with a scrolling bar indicator as would have been visible in 3D Slicer. Subtle changes in the lesions prevented an early diagnosis of this malignancy study. Subsequent to the last scan, the patient underwent wedge rejection surgery and the 
lesion was determined to be malignant. The user selects multiple slice windows in order to make a more detailed visual evaluation. The simultaneous visualization of multiple lesion CT images by the virtual reality application could assist in the detection of subtle changes in the structure of the solid component of the lesion, thereby confirming the malignant status at an earlier stage. We found the tasks of identification and classification of the lesion to be generally accomplishable albeit with display resolution as a potentially limiting factor especially for cases with small and easily missed important details. This may be resolved with improvements from further iterations of the Oculus Rift and similar devices.

We also recorded lateral head motion data using the DK2's sensors in order to visualize the amount of virtual screen area that is being used in order to conduct tasks. As shown in Figure 4.2, a negative angle corresponds to the user looking towards the left whereas a positive angle corresponds to the user looking towards the right. The data from the session depicted in the storyboard is plotted in Figure 4.3. Approximately a total of 200 degrees of lateral head motion was recorded with time spent viewing each arrayed image showing full usage of the system to make comparisons between the $\mathrm{CT}$ images. An experiment evaluating this use case is described in chapter 5.1. 


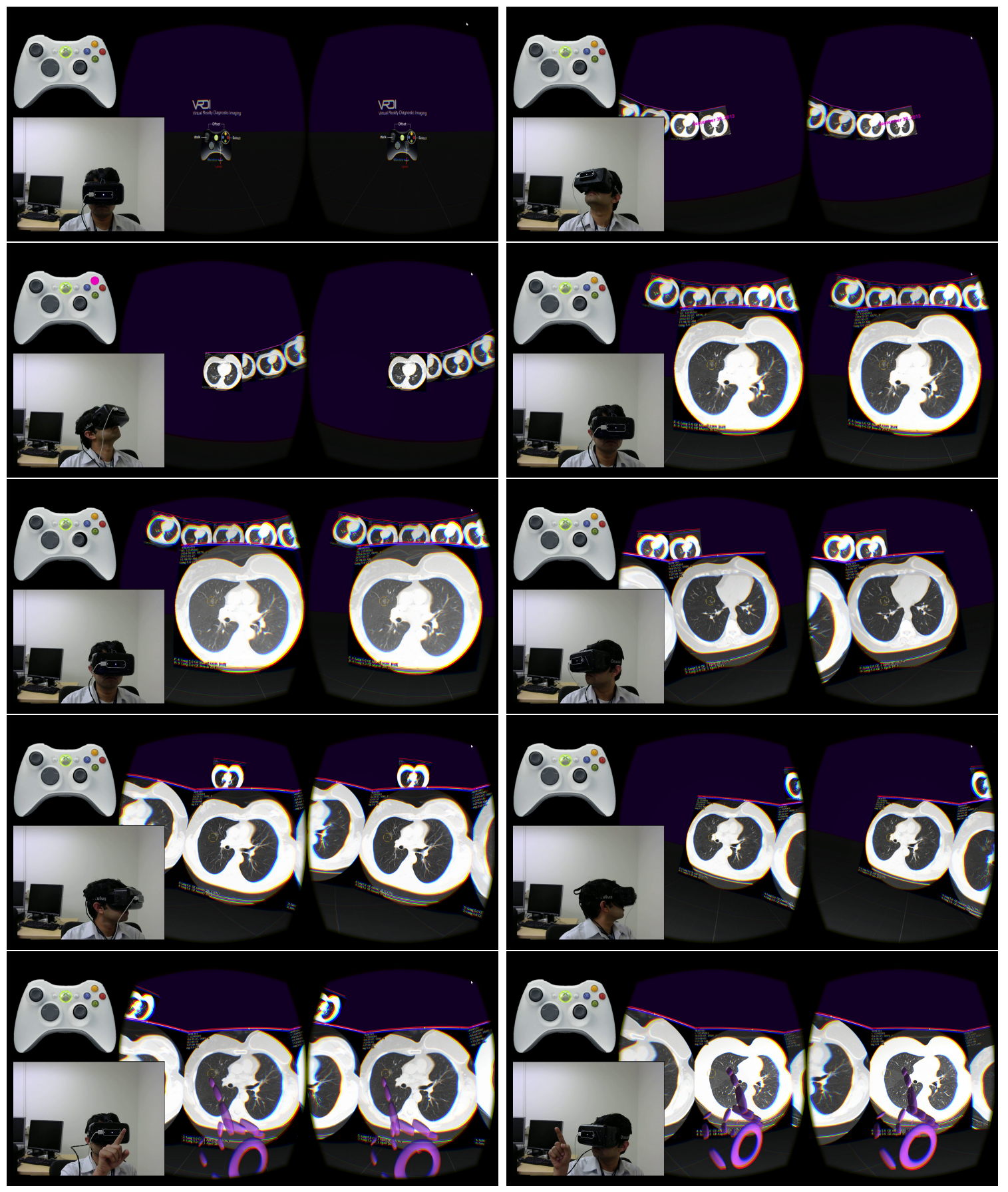

Figure 4.1: Storyboard of a session consisting of identifying a lesion on a CT lung image and evaluating its growth in a longitudinal study 


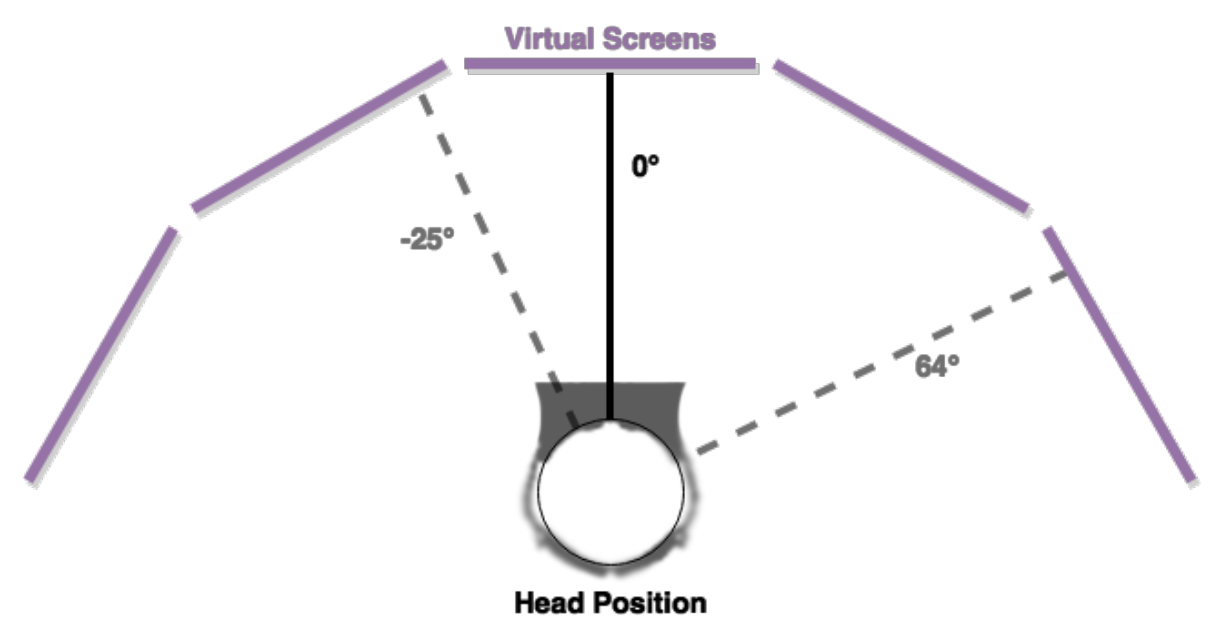

Figure 4.2: Definition of lateral head facing for Figure 4.3

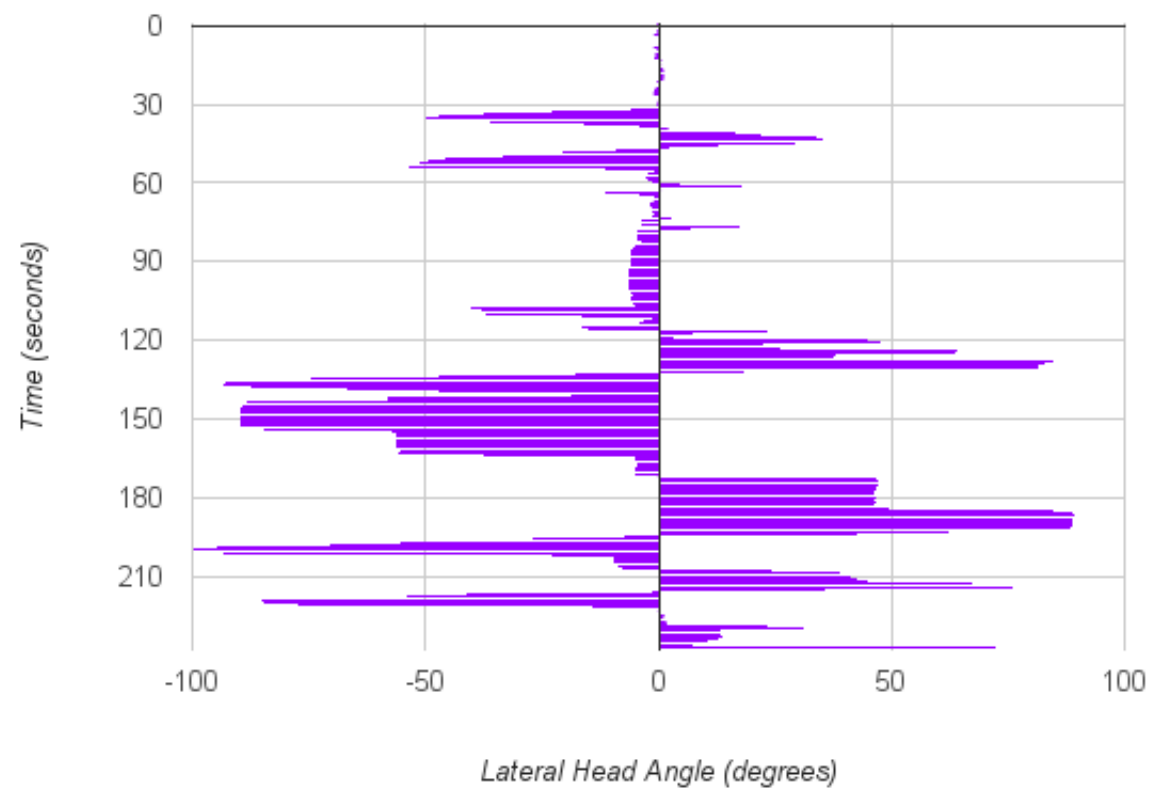

Figure 4.3: Recorded lateral head facing over the course of the storyboard session 


\subsection{Remote Instruction and Collaboration}

Taking advantage of multi-user master and slave functionality the virtual reality system was used as an instructional teaching tool as well. As seen in Figure 4.4 the master user was able to select specific image datasets out of those loaded and, using the mounted Leap Motion controller, was able to perform a hands-on demonstration of lesion detection. An experiment evaluating this use case is described in chapter 5.2 .

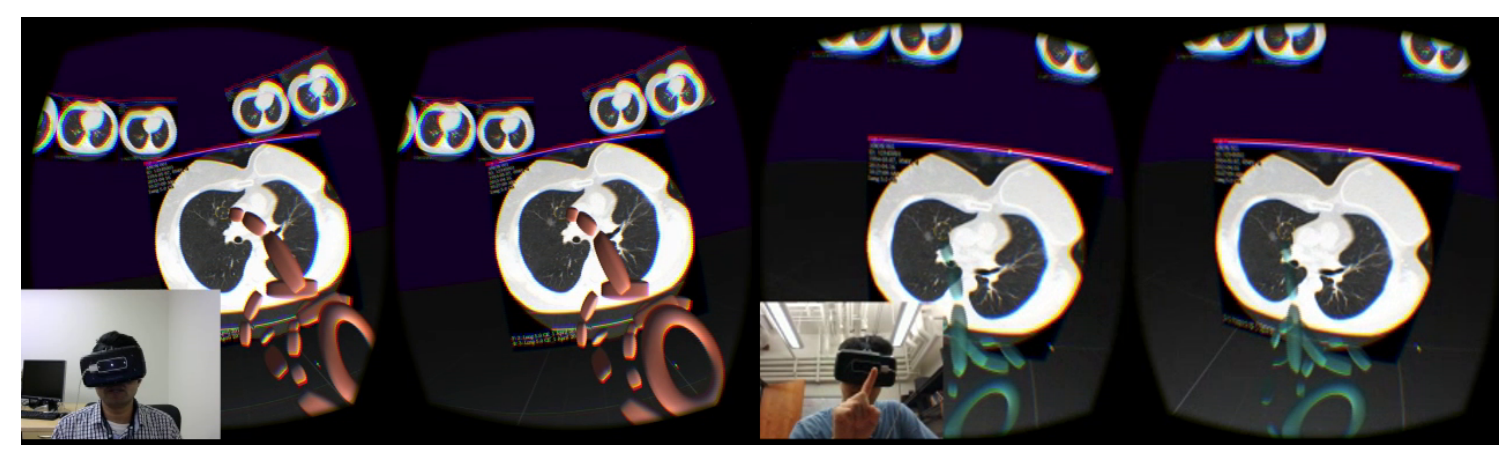

Figure 4.4: Master user locates a lesion and highlights it with a finger

\subsection{Visualizing Large Datasets}

The system may also be used for the visualization of a large amount of dataset in order to allow for quick overall comparisons to be made. Monitoring of disease activity in multiple sclerosis is typically accomplished using MRI imaging of lesions that form on the brains of patients [27]. Resulting MRI time-series studies may span the course of months generating enough image data that it is beneficial to simultaneously view multiple MRI volumes at once in order to track disease progression. Shown in Figure 4.5 is usage of the prototype for displaying a temporal MRI series consisting of 21 MRI volumes for a multiple sclerosis patient. The windows fill a large portion of the 
field of view of a user and lesion changes are visible across the dataset allowing for quick overall comparisons and evaluation of lesion evolution to be made. As with the previous case, multiple images were selected in the event detailed comparisons needed to have been made. An experiment was not yet conducted for this use case and is left for future work.

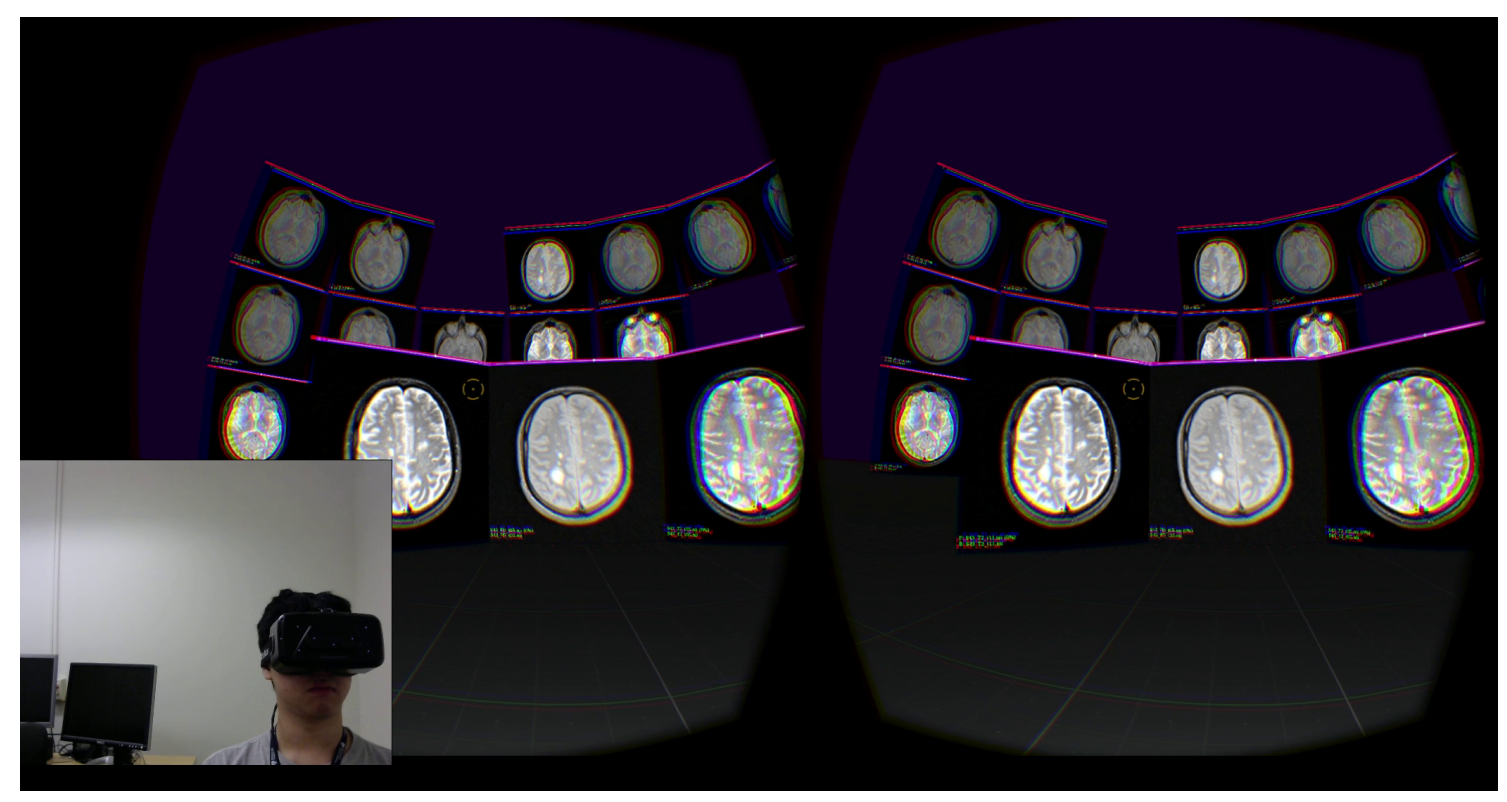

Figure 4.5: Visualization of a large number of image datasets 


\section{Chapter 5}

\section{EXPERIMENTS AND RESULTS}

\subsection{Differential Diagnosis Experiment}

In this section we describe the design and results of an experiment evaluating usage of the virtual reality diagnostic imaging system for differential diagnosis, specifically for lung lesion identification and assessment, as was described in Chapter 4.1.

\subsubsection{Differential Diagnosis Experiment Design}

A trial was conducted in July 2015 with the intention of determining the efficacy of the virtual reality diagnostic imaging prototype in allowing a clinician to both identify lesions and make a determination about growth of a lesion or its solid component. Growth of a lesion is a predictor of malignancy [31] and the identification and growth determination of lesions is useful for tracking disease progression and determining treatment response for known tumors. The tasks presented to participating radiologists were designed with the assistance of a radiologist to be a simplified representation of radiological tasks.

Five radiologists (4 attending and 1 resident) from Brigham and Women's Hospital 
were recruited to participate in a pilot study. Twenty lung CT datasets were obtained consisting of cases with nodules, including ground glass opacity, and 3 or more comparison studies. Participants were given two tasks to perform on each dataset. First, participants were asked to locate the largest lung lesion within each image series. Second, participants were asked to locate the lesion on each image within the dataset and make an assessment as to whether the lesion had grown by qualitatively comparing each image. We predetermined the largest lesion within each image series and its growth status using diameter and cross-sectional area measurements (Figure 5.1) establishing a ground truth. Datasets were qualitatively categorized as either easy, moderate, or hard primarily based on difficulty of identifying the largest lesion and secondarily based on the difficulty of making an assessment about its growth. For each radiologist, 10 datasets were randomly assigned to be read using the virtual reality diagnostic imaging system whereas the other 10 datasets were read using RadiAnt ${ }^{1}$, a free-to-use DICOM viewer.

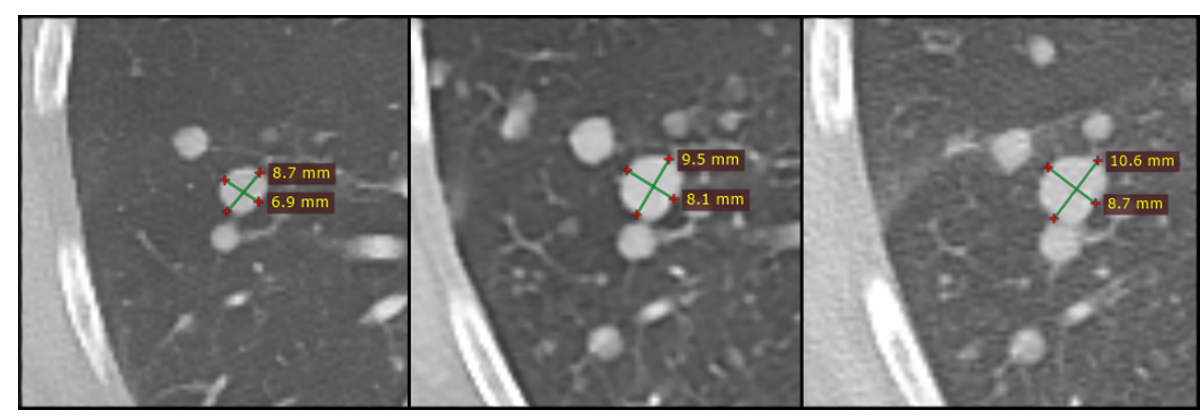

Figure 5.1: Diameter and cross-sectional measurements of lung lesions

Participants assigned to use the virtual reality diagnostic imaging system first were given an explanation of the task to be performed and instruction in the operation of the system. Participants were then fitted with the Oculus Rift DK2 virtual reality

\footnotetext{
${ }^{1}$ http://www.radiantviewer.com/
} 
head-mounted display. The VR system was then started with an instructional image showing allowing for the participants to adjust the headset until an optimal focus and clarity was achieved. Once the participant was comfortable, a random dataset was loaded into the system and displayed. The participant would then select the most recent image in the dataset and locate a nodule. Time taken from the moment the button was pressed for the selection of the image to when the participant could highlight the location of a nodule with the reticule was recorded. Participants continued to select the other images in the dataset locating the nodule in each. With all the images selected, each participant would then perform a comparison of the images in order to determine its growth status. The option to deselect and push back images to view fewer at a time was given as well. The overall time taken to determine growth status of the lesion was also recorded. A new dataset was then loaded and the procedure was repeated for all 10 datasets that were randomly assigned to the VR system. Afterwards, participants were asked to complete a NASA Task Load Index (TLX) questionnaire [15] designed to rate perceived workload resulting from using the system (see Appendix A for questionnaire). Both tasks were then repeated using RadiAnt and another TLX questionnaire was given. Comments made by participants were recorded throughout the experiment.

\subsubsection{Differential Diagnosis Experiment Results}

In total, 4 participants assessed 20 image datasets for a total of 80 assessments between the VR system and a standard DICOM viewer. Table 5.1 shows the number of datasets assessed by each method and the number of assessments of growth status of each that were consistent with the ground truth. 
Table 5.1: Total number of cases assessed by all participants and assessments that were consistent with measured results

\begin{tabular}{lrrr|rrr} 
& \multicolumn{3}{c|}{ Virtual Reality System } & \multicolumn{3}{c}{ Standard DICOM Viewer } \\
\hline & Easy & Moderate & Hard & Easy & Moderate & Hard \\
\hline Correct Assessments & 10 & 12 & 22 & 10 & 12 & 20 \\
Total Cases & 10 & 15 & 25 & 10 & 15 & 25 \\
\hline & $100 \%$ & $80 \%$ & $88 \%$ & $100 \%$ & $80 \%$ & $80 \%$ \\
\hline
\end{tabular}

No significant difference $(p>0.05)$ in the overall success rate of lesion identification and classification was observed between the VR system and a standard DICOM viewer.

A significant difference between systems was found (Student's $t$-test, $p<0.05$ ) in the average time taken to identify the largest lesion. Identification time separated by difficulty rating can be seen in Figure 5.2. It was noted that the difference in time taken for the identification of lesions classified as hard was not significant $(p>0.05)$.

As was the case with lesion identification time, a significant difference between systems for the task of assessing the presence of lesion growth was found (Student's $t$-test, $p<0.01)$, however, the difference again was not significant $(p>0.05)$ when comparing only lesions classified as hard to asses. A breakdown by lesion difficulty rating is seen in Figure 5.3.

There was a significant effect on system type on task load (repeated measures ANOVA for 2 systems and 6 task load dimensions, $p<0.01$ ). Usage of the VR system for lesion identification and assessment resulted in greater task load when compared to usage of a standard DICOM viewer.

The average range of lateral head motion across all datasets and participants was 


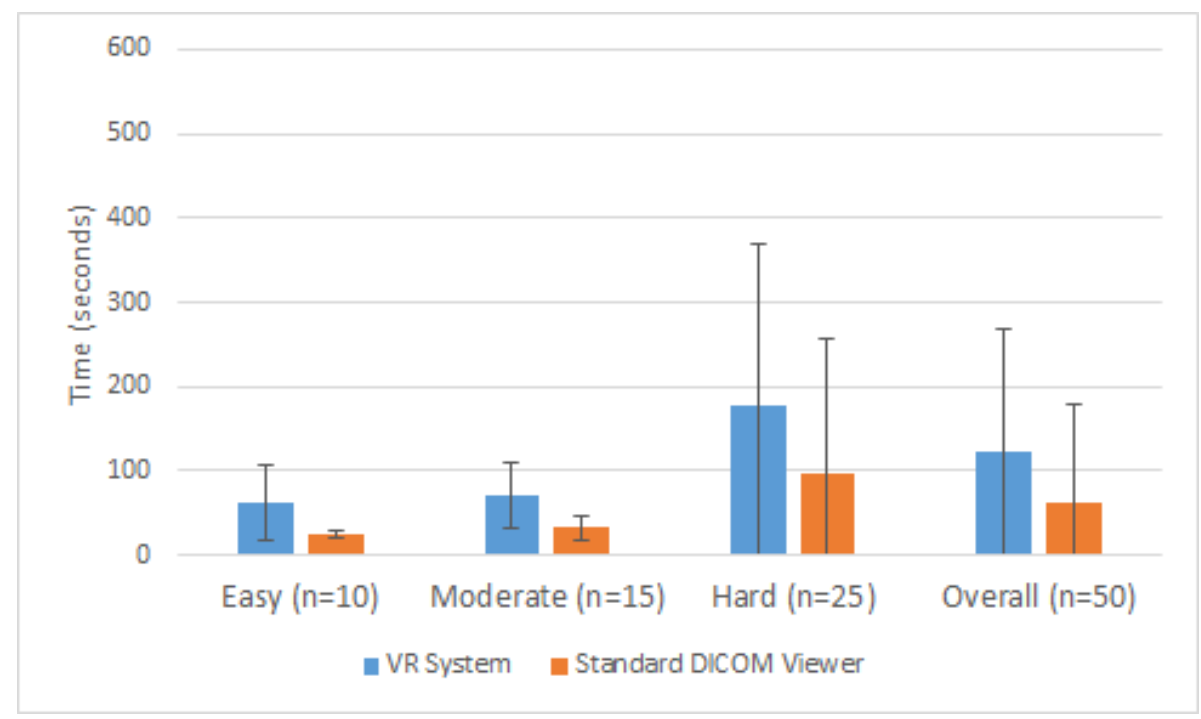

Figure 5.2: Average time taken for identification of the largest lung lesion per dataset. Error bars are standard deviation of the mean.

$144 \pm 45$ degrees (standard deviation).

Comments made by some participants indicated discomfort with the weight of the DK2 and the necessity of physically adjusting the DK2 until the image was in focus. There were also comments indicating frustration with the speed of scrolling through images using the gamepad controller.

\subsubsection{Differential Diagnosis Experiment Discussion}

Despite differences in task load and time taken, the success rate of both systems were virtually identical. Differences in time taken may be explained by the specific interface used for the VR system. Although a gamepad controller may have been more suitable for a VR environment, joystick and trigger usage may reduce a user's precision in scrolling through a slice and jumping to specific positions. Comments made by participants suggested that a faster scroll speed may have been preferred by participants which may have led to faster completion of tasks. Slower scrolling 


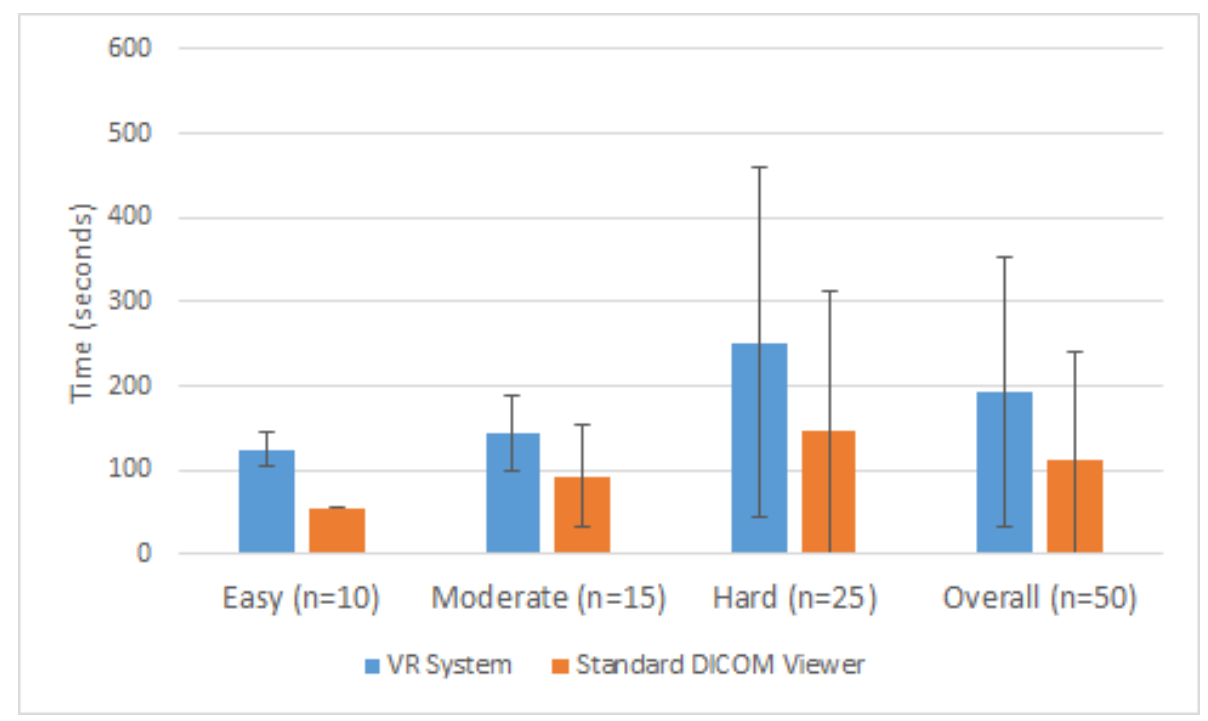

Figure 5.3: Average time taken for growth assessment of the largest lung lesion per dataset. Error bars are standard deviation of the mean.

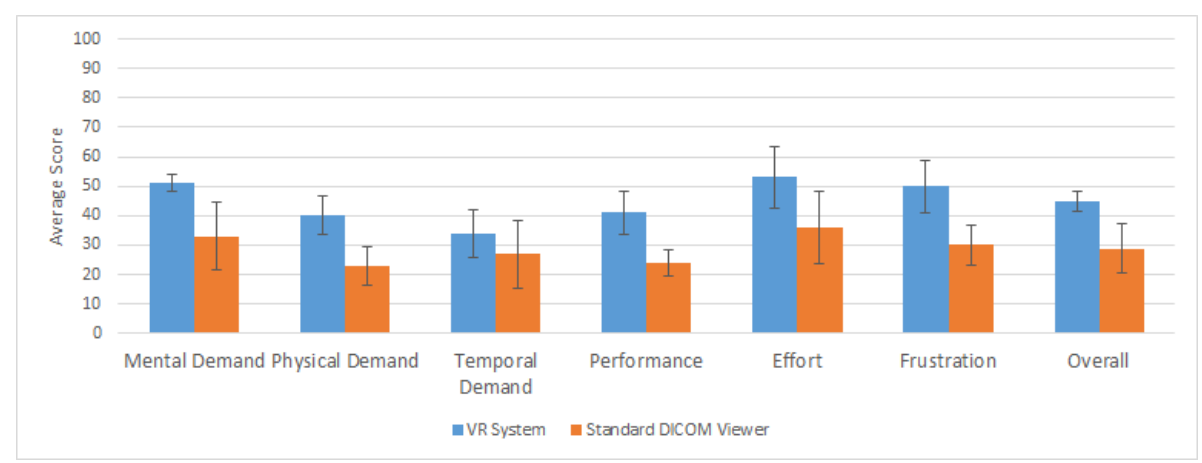

Figure 5.4: NASA TLX for VR and standard DICOM viewer usage. Error bars are for standard error.

and image selection could result in a consistently greater time required while time for decision making and visual assessment is reduced or unchanged. The discomfort resulting from the need to strap a physical device to one's head may account for the increased task load for usage of a VR system. Additionally, radiologists would likely be more familiar with using a standard DICOM viewer than a VR system.

An additional factor that may have influenced the greater task load of using 
the VR system was VR motion sickness. One participant reported discomfort after extended use of the DK2 while performing the lesion identification tasks. One theory as to the cause of VR motion sickness is that it is the result of conflicting sensory input from the visual system and vestibular system [2]. The VR system designed and used for the experiments allowed users to physically walk within the virtual environment and some participants did make use of the feature. Restricting this functionality may serve to reduce the severity of the conflict between differing sensory inputs and overall serve to reduce the task load of using the VR system for radiological tasks. Walking functionality may be replaced with increased control of the overall environment such as allowing for rotation of image datasets around the user. Other considerations in regards to VR motion sickness include refresh rate, latency, duration of a session, and a user's personal experience with VR environments [35]. Many of these other considerations can be accounted for with improvements to specifications of future VR devices.

The results of the pilot study suggest that a VR system can be used to achieve equal results for certain radiological tasks as what would otherwise be done with PACS or a standard monitor-based DICOM viewer. Although the success of the task was identical between systems, subjective comments made by participants suggested that numerous virtual displays may have been helpful in solidifying decisions made about growth assessment. The limited number of participants warrants future studies investigating in further detail the potential benefits of numerous virtual displays, especially with a greater focus on datasets with a large number of images. Additionally, expanding the use of VR for radiology beyond the assessment of 2D image slices should be studied further. The immersive stereoscopic environment provided 
by VR devices could assist in volume measurement of lesions as an alternative to cross-section and diameter measurements [17].

\subsection{Remote Collaboration Experiment}

In this section we describe the design and results of an experiment evaluating usage of the virtual reality diagnostic imaging system for basic remote collaboration and teleradiology tasks as was described in Chapter 4.3.

\subsubsection{Remote Collaboration Experiment Design}

After the lesion identification experiment, each participant was asked to take part in a remote collaboration experiment. The VR system was loaded with an unused dataset and the participant was once again fitted with the DK2 headset. A master computer was set up in a remote room with data loaded from the same 3D Slicer instance. The participant's computer then connected to the master computer allowing for synchronized viewing of image datasets, hand position, and the gaze of the master user represented with a circular reticule. The master user then proceeded to direct their gaze to 5 anatomical features within the image series each time asking the participant to point at the feature with their hand and verbally confirm the identity of the anatomical feature (see Appendix A for data sheet). Afterwards, the participant completed another TLX questionnaire.

\subsubsection{Remote Collaboration Experiment Results}

Each participant was successfully able to pinpoint and name every anatomical figure indicated by the master user. The task load caused by the task (5.5) was low in 
comparison to identification and assessment tasks.

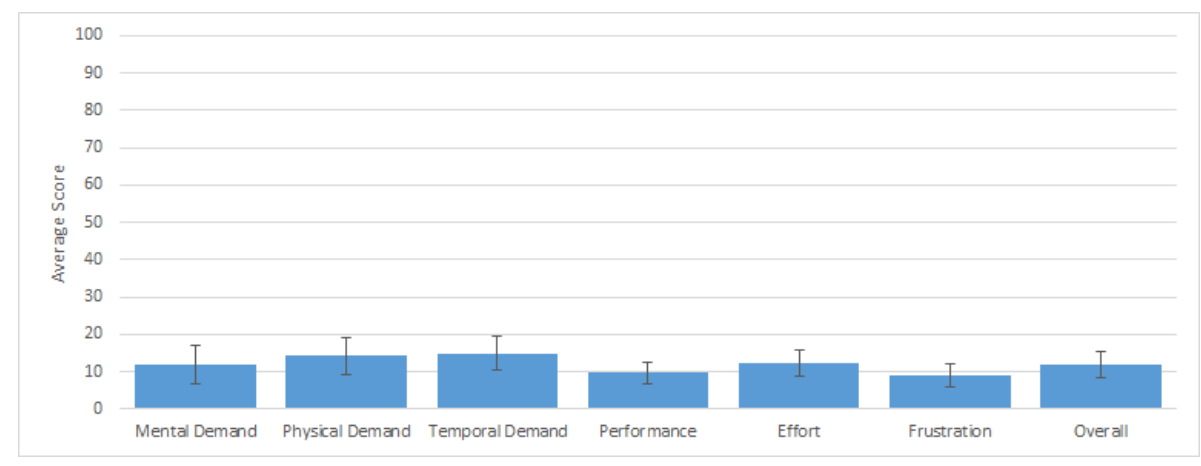

Figure 5.5: NASA TLX for remote collaboration. Error bars are for standard error.

\subsubsection{Remote Collaboration Experiment Discussion}

The results of the remote collaboration phase of the experiment suggest that the multiuser functionality of the VR system is capable of enabling basic radiological tasks. In addition to the technical aspect of synchronizing remote movements and gaze direction markers, the image quality of the DK2 display was sufficient enough for participants to recognize multiple anatomical features. The task load required as a passive user of the VR system was also reduced in comparison to requiring a user to directly manipulate datasets. This may allow for numerous users who are inexperienced with VR environments to comfortably use the system and still contribute to collaboration while more experienced operators are relied upon for more involved interactions.

The experiment conducted was limited only to remote radiological collaboration although remote multidisciplinary collaboration could also be facilitated by a VR system. 


\section{Chapter 6}

\section{CONCLUSIONS AND FUTURE WORK}

\subsection{Summary of Work}

In this work we examined immersive virtual reality devices especially those targeted towards the consumer market with an emphasis on cost and portability. We highlighted certain advantages that consumer virtual reality devices can offer diagnostic imaging such as scalable virtual screen number, remote collaboration potential, decreased usage of space, and cost.

We developed a VR environment intended to act as a virtual radiology reading room that allowed users to load and manipulate medical images. The use of a virtual environment enabled users to take advantage of a wide virtual viewing area that may have been unfeasible to replicate in physical reality. We experimented with multiple interface concepts before settling on a handheld controller for the interim. Our initial experiences suggest that the image quality provided by the Oculus Rift DK2 display was sufficient to perform a differential diagnosis task with a demonstrated use case and dataset.

We evaluated the ability of attending radiologists and residents to use the virtual 
reality diagnostic imaging environment to perform radiological tasks recording the ability to identify lesions on multiple datasets, accuracy of conclusions made about nodule growth, the time taken to perform the tasks, and the overall physical and mental demand reported by the participants. Furthermore, we evaluated the ability of the virtual reality environment to act as a remote collaboration and instructional tool where participants underwent the task of identifying structures marked by an instructing user.

\subsection{Future Work}

The focus of future work is on extending the applications of virtual reality within radiology. Usage of the system created for this work was limited to replicating a radiology reading room environment primarily for $2 \mathrm{D}$ images. Although the interpretation of 3D volumes has comparatively less importance within the field of radiology than 2D images, the introduction of cheap stereoscopic virtual reality displays may prove to improve the desirability for 3D volume visualization.

Beyond radiology there exist numerous potential applications opened up by affordable consumer virtual reality. In addition to image datasets, real-time 3D video from stereo endoscopes and other imaging devices can be made available for laparoscopic procedures. Virtual reality may also provide an intuitive method for navigation with virtual endoscopy or viewing and assisting in interpretation of diffusion tensor imaging by way of tractography visualization.

New iterations of consumer virtual reality devices offer improvements in image quality and field of view and additional features such as eye-tracking. New interface

devices focused on interaction within an immersive virtual environment also improve 
the range of tasks available to users. Another strong focus of future work is following these advancements in consumer virtual reality and applying them to medical imaging.
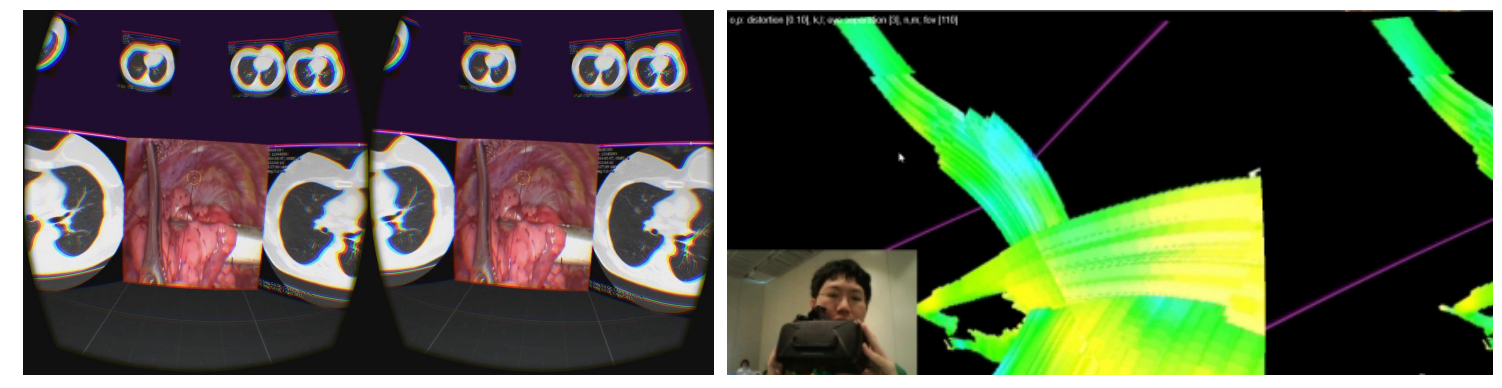

Figure 6.1: Stereoscopic video support (left) Tractography fiber bundles viewed in virtual reality (right) 


\section{Bibliography}

[1] Z. Ai, F. Dech, M. Rasmussen, and J. C. Silverstein. Radiological tele-immersion for next generation networks. Stud Health Technol Inform, 70:4-9, 2000.

[2] H. Akiduki, S. Nishiike, H. Watanabe, K. Matsuoka, T. Kubo, and N. Takeda. Visual-vestibular conflict induced by virtual reality in humans. Neuroscience Letters, 340(3):197 - 200, 2003.

[3] R. Ball and C. North. Effects of tiled high-resolution display on basic visualization and navigation tasks. In CHI 2005 Extended Abstracts on Human Factors in Computing Systems, pages 1196-1199, 2005.

[4] M. T. Bolas. Human factors in the design of an immersive display. Computer Graphics and Applications, IEEE, 14(1):55-59, Jan 1994.

[5] S. Bryson. Virtual reality in scientific visualization. Commun. ACM, 39(5):6271, May 1996.

[6] C. J. Calvano, M. E. Moran, L. D. Tackett, P. P. Reddy, K. E. Boyle, and M. M. Pankratov. New visualization techniques for in utero surgery: amnioscopy with a three-dimensional head-mounted display and a computer-controlled endoscope. J. Endourol., 12(5):407-410, Oct 1998. 
[7] Leap Motion Controller. https://www.leapmotion.com/.

[8] Microsoft Xbox 360 Controllers. http://www.xbox.com/en-US/xbox-360/ accessories/controllers.

[9] C. Cruz-Neira, D. J. Sandin, and T. A. DeFanti. Surround-screen projectionbased virtual reality: The design and implementation of the CAVE. In Proceedings of the 20th Annual Conference on Computer Graphics and Interactive Techniques, SIGGRAPH '93, pages 135-142, New York, NY, USA, 1993. ACM.

[10] M. Czernuszenko, D. Pape, D. Sandin, T. DeFanti, G. L. Dawe, and M. D. Brown. The ImmersaDesk and Infinity Wall projection-based virtual reality displays. SIGGRAPH, 31(2):46-49, 1997.

[11] Unity Game Engine. https://unity3d.com/.

[12] A. Fedorov, R. Beichel, J. Kalpathy-Cramer, J. Finet, J-C. Fillion-Robin, S. Pujol, C. Bauer, D. Jennings, F. Fennessy, M. Sonka, J. Buatti, S. R. Aylward, J. V. Miller, S. Pieper, and R. Kikinis. 3d Slicer as an image computing platform for the quantitative imaging network. Magn Reson Imaging, 30(9):1323-1341, 2012.

[13] S. S. Fisher, M. McGreevy, J. Humphries, and W. Robinett. Virtual environment display system. In Proceedings of the 1986 Workshop on Interactive 3D Graphics, I3D '86, pages 77-87, New York, NY, USA, 1987. ACM.

[14] F. Garzotto and M. Valoriani. "Don't touch the oven": Motion-based touchless interaction with household appliances. In Proceedings of the International Working Conference on Advanced Visual Interfaces, AVI '12, pages 721-724, New York, NY, USA, 2012. ACM. 
[15] S. G. Hart and L. E. Staveland. Development of NASA-TLX (task load index): Results of empirical and theoretical research. In Human Mental Workload, volume 52 of Advances in Psychology, pages 139 - 183. North-Holland, 1988.

[16] J. G. Hincapié-Ramos, X. Guo, P. Moghadasian, and P. Irani. Consumed endurance: A metric to quantify arm fatigue of mid-air interactions. In Proceedings of the 32Nd Annual ACM Conference on Human Factors in Computing Systems, CHI '14, pages 1063-1072, New York, NY, USA, 2014. ACM.

[17] S. G. Jennings, H. T. Winer-Muram, R. D. Tarver, and M. O. Farber. Lung tumor growth: Assessment with CTcomparison of diameter and cross-sectional area with volume measurements. Radiology, 231(3):866-871, 2004. PMID: 15163822.

[18] N. Kothary, L. Lock, D. Y. Sze, and L. V. Hofmann. Computed tomographyguided percutaneous needle biopsy of pulmonary nodules: Impact of nodule size on diagnostic accuracy. Clin Lung Cancer, 10(5):360-363, Sep 2009.

[19] Christian Krapichler, Michael Haubner, Rolf Engelbrecht, and Karl-Hans Englmeier. VR interaction techniques for medical imaging applications. Computer Methods and Programs in Biomedicine, 56(1):65 - 74, 1998.

[20] B. Laurel. Computers As Theatre. Addison-Wesley Longman Publishing Co., Inc., Boston, MA, USA, 1991.

[21] P. Milgram, H. Takemura, A. Utsumi, and F. Kishino. Augmented reality: a class of displays on the reality-virtuality continuum, 1995.

[22] Thalmic Labs Myo. https://www.thalmic.com/myo/rl. 
[23] P. Nagy, E. Siegel, T. Hanson, L. Kreiner, K. Johnson, and B. Reiner. PACS reading room design. Seminars in Roentgenology, 38(3):244 - 255, 2003. Digital Imaging.

[24] D. C. Neale. Head-mounted displays: Product reviews and related design considerations. In Considerations, Hypermedia Technical Report HCIL-98-02, HumanComputer Interaction Laboratory, Department of Industrial and Systems Engineering, Virginia Tech, 1998.

[25] T. Ni, D. A. Bowman, and J. Chen. Increased display size and resolution improve task performance in information-rich virtual environments. In Proceedings of Graphics Interface, pages 139-146, 2006.

[26] S. Pieper, M. McKenna, D. Chen, and I. McDowall. Computer animation for minimally invasive surgery: Computer system requirements and preferred implementations. In Proceedings of SPIE 2177 Stereoscopic Displays and Virtual Reality Systems, pages 401-408, 1994.

[27] A. Rovira, C. Auger, and J. Alonso. Magnetic resonance monitoring of lesion evolution in multiple sclerosis. Ther Adv Neurol Disord, 6(5):298-310, Sep 2013.

[28] L. L. Rumreich and A. J. Johnson. From traditional reading rooms to a soft copy environment: Radiologist satisfaction survey. Journal of Digital Imaging, 16(3):262-269, 2003.

[29] E. Siegel, B. Reiner, M. Abiri, A. Chacko, R. Morin, D. W. Ro, K. Spicer, N. Strickland, and J. Young. The filmless radiology reading room: A survey of 
established picture archiving and communication system sites. Journal of Digital Imaging, 13(1):22-23, 2000.

[30] I. E. Sutherland. A head-mounted three dimensional display. In Proceedings of AFIPS, pages 757-764, 1968.

[31] S. J. Swensen, M. D. Silverstein, D. M. Ilstrup, C. D. Schleck, and E. S. Edell. The probability of malignancy in solitary pulmonary nodules: Application to small radiologically indeterminate nodules. Archives of Internal Medicine, 157(8):849$855,1997$.

[32] F. Tyndiuk, V. Lespinet-Najib, G. Thomas, and C. Schlick. Impact of large displays on virtual reality task performance. In Proceedings of AFRIGRAPH 2004, pages 61-65, 2004.

[33] Oculus VR. https://www.oculus.com/.

[34] B. G. Witmer and M. J. Singer. Measuring presence in virtual environments: a presence questionnaire. Presence: Teleoperators and Virtual Environments, 7(3):225-240, 1998.

[35] R. Yao, T. Heath, A. Davies, T. Forsyth, N. Mitchell, and P. Hoberman. Oculus VR best practices guide. Oculus VR, 2014. 


\section{Appendix A}

\section{Trial Data Forms}

\section{A.1 NASA Task Load Index Questionnaire}

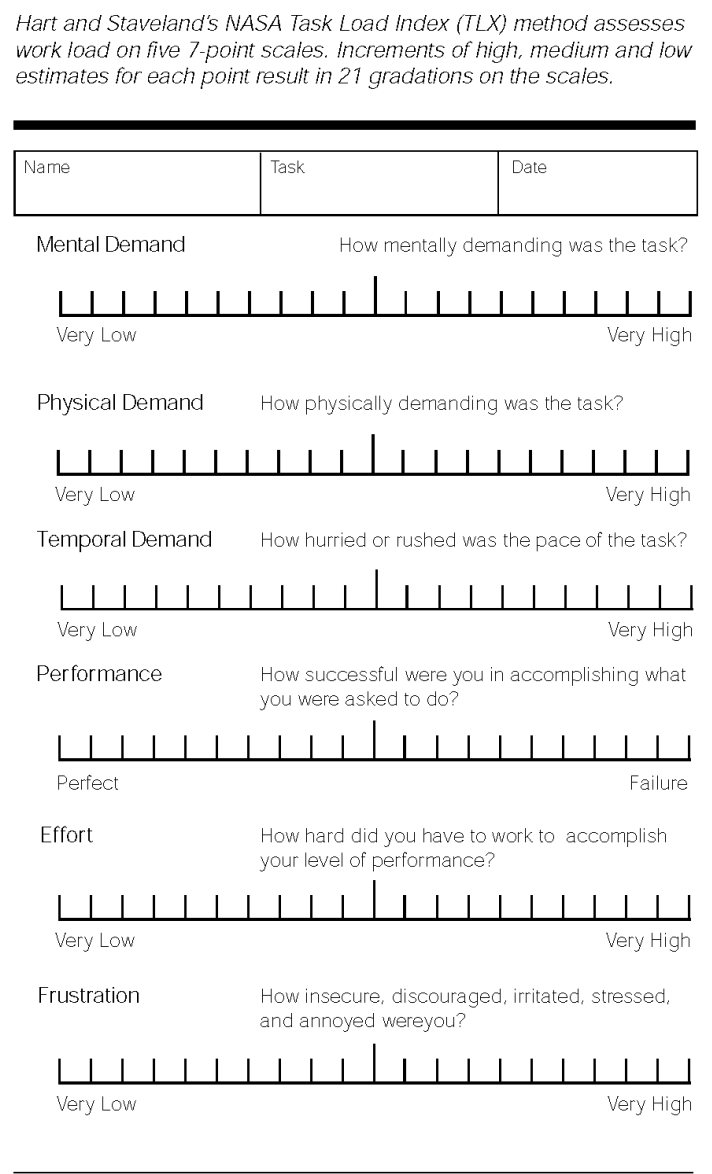




\section{A.2 Data Sheet}

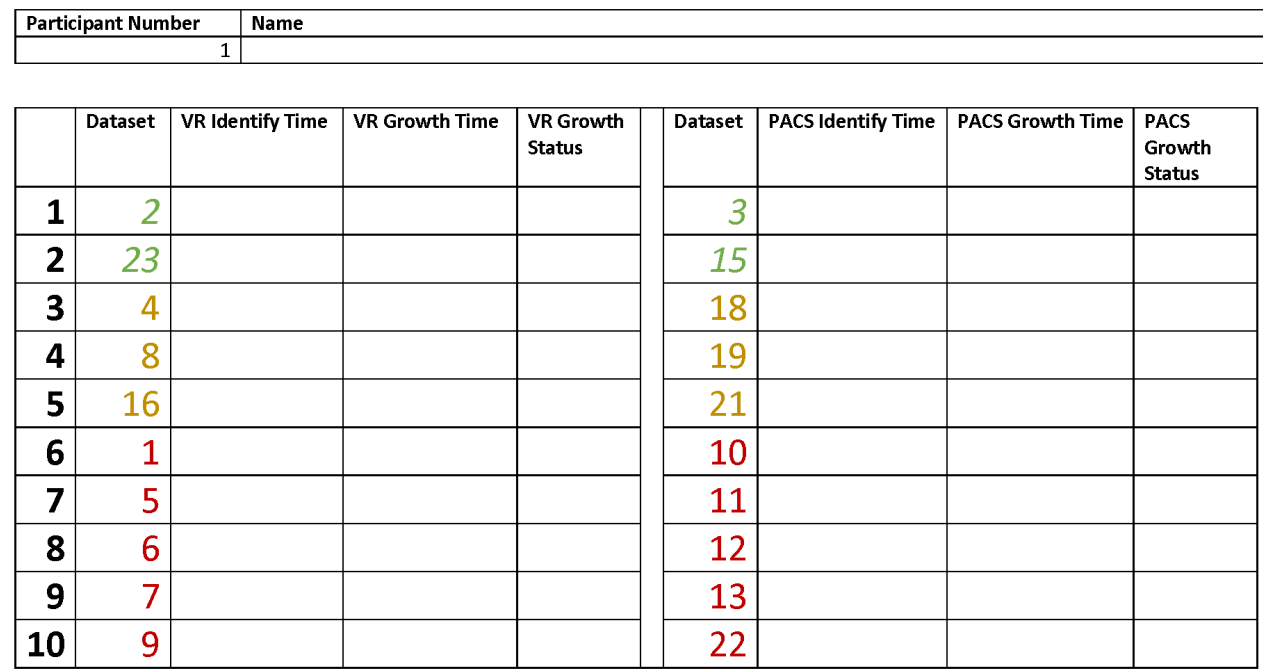

\begin{tabular}{|l|l|}
\hline Collaboration Feature & Identified \\
\hline Lesion & \\
\hline Implant & \\
\hline Aorta & \\
\hline Pulmonary Vein & \\
\hline Splenic Artery/Vein & \\
\hline
\end{tabular}

